\title{
Deep subwavelength Fabry-Perot resonances
}

\author{
Cheng-Ping Huang ${ }^{1,2, *}$ and Che-Ting Chan ${ }^{1, \mathrm{a}}$ \\ 1 Department of Physics, The Hong Kong University of Science and Technology, Clear Water Bay, Kowloon, Hong Kong, P.R. China \\ 2 Department of Applied Physics, Nanjing Tech University, Nanjing 210009, P.R. China12
}

Received 10 April 2014 / Accepted 01 May 2014

\begin{abstract}
Confinement of light by subwavelength objects facilitates the realization of compact photonic devices and the enhancement of light-matter interactions. The Fabry-Perot (FP) cavity provides an efficient tool for confining light. However, the conventional FP cavity length is usually comparable to or larger than the light wavelength, making them inconvenient for many applications. By manipulating the reflection phase at the cavity boundaries, the FP cavity length could be made much smaller than the wavelength. In this review, we consider the subwavelength FP resonance in a plasmonic system composed of a slit grating backed with a ground plane, covering the spectral range from microwave to $\mathrm{THz}$ and infrared regime. For very narrow slit width and spacer thickness, a typical zero-order and deep subwavelength FP resonance in the metallic slits can be strongly induced. Moreover, due to the subwavelength FP resonance, greatly enhanced electromagnetic pressure can also be induced in the system. The sign and magnitude of the electromagnetic pressure are dominated by the field penetration effect in the metal as well as the field enhancement in the FP cavities. The effect promises a variety of potential applications, such as detecting tiny motions and driving the mechanical oscillations.
\end{abstract}

Key words: Plasmonics, Subwavelength, Fabry-Perot cavity, Resonance.

\section{Introduction}

Waves in the periodic media are subject to the Bragg scattering, giving rise to a band structure. Such effect presents in both the electronic and photonic or phononic crystals [1-4], where the lattice constants are of the order of the wavelength. For waves with a long wavelength, the manipulation of wave motion typically requires a bulky device. On the other hand, by utilizing the strong resonance effect, waves can also be manipulated by the subwavelength or even deep-subwavelength objects. One example is the piezo- electric superlattice composing of periodic ferroelectric domains [5-7], where the coupling between the electromagnetic wave and superlattice vibration may lead to a polaritonic bandgap in the long-wavelength region. Another example is the so-called "locally resonant sonic material" $[8,9]$, which can block acoustic waves with wavelength much larger than the characteristic size of the crystal. Recently, plasmonic materials have attracted much research interests $[10,11]$. It is well known that the plasmonic resonance of nanostructures can be employed to confine light or guide the flow of light $[12,13]$. Moreover, the electric and/or magnetic resonances in metamaterials with the subwavelength components have been demonstrated to enable exotic wave manipulation properties such as the negative refraction and superlensing

\footnotetext{
*e-mail: cphuang@njtech.edu.cn

a phchan@ust.hk
}

[14-16]. The concept of resonance may provide a way for miniaturizing the devices and realizing novel optical effects with subwavelength components.

There are many different resonators which can be employed to realize optical resonances, and examples include metallic nanoparticles, metallic or dielectric microdisks, and photoniccrystal cavities [12, 17-19]. Here we would like to discuss two typical optical resonators: the inductance-capacitance (LC)-like resonator and the the Fabry-Perot (FP)-like resonator. A well-known example of LC resonator is the metallic split-ring resonator (SRR), which plays a crucial role in metamaterial design [14, 20-22]. The LC resonator has an effective inductance and capacitance, inducing the free electrons to oscillate like mechanical harmonic oscillators [23]. The resonance effect can be extended from the microwave band to $\mathrm{THz}$ and infrared range by reducing the resonator sizes $[21,22]$. For example, the optical properties of nano-metals or flat metal surfaces have been studied using the LC model [24-27]. However, the scaling of resonance frequency with size will break down in the optical regime [28], due to the dominance of internal inductance. The LC resonance can be excited by the electric or magnetic field of light or by the neighboring resonators via the near-field coupling. The coupling between the LC-like resonance and electric- dipole mode can mimic the electromagneticallyinduced transparency [29].

In contrast, the FP-like resonator is an optical cavity based on the electromagnetic interference effect [30, 31]. 
A conventional FP resonator consists of an air cavity of length $L$ sandwiched between two parallel reflecting mirrors. The constructive or destructive interference of forward and backward propagating waves results in the formation of a standing wave pattern in the cavity. The FP cavity has been widely used in applications such as the laser cavity, signal filtering, directive emission, and optomechanical coupling [31-33]. However, compared with the LC-like resonator which is frequency selective and can be deep subwavelength in size, the FP-like resonator operates with the wavelength that scales with the cavity length. The resonance condition of a conventional FP cavity requires that the total phase shift of one round trip equals a multiple of $2 \pi$, i.e., $4 \pi n L / \lambda+\varphi_{1}+\varphi_{2}=2 \pi m$ (here, $n$ is the index of cavity filling medium, $\varphi_{1}$ and $\varphi_{2}$ are the reflection phases, and $m$ is an integer). Generally speaking, the reflection phase at the interface between two media has two possible values 0 or $\pi$, depending on the ratio of medium refractive index. Thus, it can be inferred that the cavity length must be comparable to or larger than the resonance wavelength. This makes the device unsuitable for applications that require small formfactor components.

In recent years, the FP-like effect in plasmonic circumstances has received much attention [34-41]. It was found that the FP-like resonances in the subwavelength apertures of a metal film can increase the transmission of light [34, 35]. Moreover, a simple plasmonic FP cavity can be constructed by using the plasmonic mirrors and/or employing the plasmonic confinement $[36,38]$. Although the quality factor is compromised due to the Ohmic loss, the presence of plasmonic effect can squeeze the light wavelength and reduce the mode area drastically. Consequently, the combination of FP resonance and surfaceplasmon resonance yields an efficient localization of light and a significant enhancement of electro- magnetic fields in the cavity [36]. By inserting the metallic nanostructures into an FP cavity, one can also observe the strong coupling between the optical mode and localized plasmon mode [40, 41]. These effects may be used for increasing the optical nonlinearity or enhancing the cavity-based plasmonic sensing. Although a simple plasmonic FP cavity may provide a solution for the miniaturization of device, the fundamental limit on the cavity length cannot be circumvented in this way.

It was noted by Trentini that the FP resonators could be created with a partially reflecting reflector that induces a phase shift different from 0 or $\pi$ [42]. This causes a slight variation of cavity length with respect to the standard value. Although the effect was not very obvious in the original structure, significant advances can be achieved now thanks to the modern metamaterial designs. In 1999, Sievenpiper et al. suggested that a mushroom-like structure, consisting of an array of metal plates connected to a back metal sheet, can act as a high-impedance surface $[43,44]$. The reflection phase of such a surface may experience a transition with frequency from $\pi$ to $-\pi$ by crossing through zero at the LC resonance frequency. With this unusual reflection property, a subwavelength FP cavity with cavity length one order of magnitude smaller than the resonance wavelength has been designed and constructed operating in the microwave band [45]. By going from one to all three dimensions [46], a cubic cavity with each dimension about a quarter of the resonance wavelength was also realized. The FP cavity length can be further squeezed by using the double metamaterial-based reflectors. The reflection phases at both reflector surfaces decrease gradually with the frequency; in a narrow frequency range, the two phases cancel each other partially, allowing for an ultrathin cavity with the length as small as $\lambda / 60$ [47]. With such cavities, an ultrasmall FP antenna, i.e., a subwavelength FP cavity coupled with an internal antenna, can provide a highly directive emission at the resonance $[45,47,48]$.

The subwavelength FP cavities constructed with the multilayer metamaterial reflectors are of the complicated geometry and thus not easy to realize at high frequencies. In this review, we discuss the designs and properties of a special kind of subwavelength FP resonator, which is composed of a metallic slit grating backed with a ground plane. Due to the unique phase shift, the metallic slits within the structure may act as "deep subwavelength" FP cavities [49], where the resonance wavelength can be two orders of magnitude larger than the cavity length. Compared with the previous designs, our FP cavity is open-ended without the microstructured physical reflectors at the cavity openings. Thus the cavity can be reduced with the nanoscale sizes and can work at high frequencies. The deep subwavelength FP-like resonance is also accompanied by many interesting physical properties [50], such as enhanced absorption and enhanced electromagnetic pressure.

A systematic discussion will be presented in the following sections. In Section 2, several mechanisms for the nontrivial phase shift at the reflecting interface are analyzed. This could be helpful for understanding Section 3, where the reflection phase and resonance condition of the proposed subwavelength FP (SFP) resonator are presented. In Section 4, we discuss the SFP resonance in the microwave band. The enhanced electromagnetic pressure due to the SFP resonance was emphasized. The effect is extended in Section 5 to the higher frequency $\mathrm{THz}$ and infrared regime. The role of the field penetration effect in the metal is also suggested. Some additional comments are provided in Section 6 and the findings are summarized in Section 7.

\section{Reflection phase at the boundaries}

\subsection{Phase shift due to resonant impedances}

For materials involving both electric and magnetic response, the reflection coefficient of light at normal incidence is determined by the impedance of the interface medium,

$$
r=\frac{Z_{1}-Z_{2}}{Z_{1}+Z_{2}}
$$

Here, $Z_{j}=\sqrt{\mu_{j} \varepsilon_{j}}$ is the impedance of a bulk medium.

For natural materials, the impedance and the phase shift are usually monotonous functions of frequency. However, by using the designed artificial materials or metamaterials with the subwavelength constituents, the electric and magnetic response can be engineered at will. For example, an array of metallic SRRs shows a magnetic resonance [20-22] near which the permeability can be enhanced, thus giving rise to higher impedance. Moreover, a negative permeability may also be induced in a frequency gap. On the other hand, an array of metallic nanoparticles has an electric 

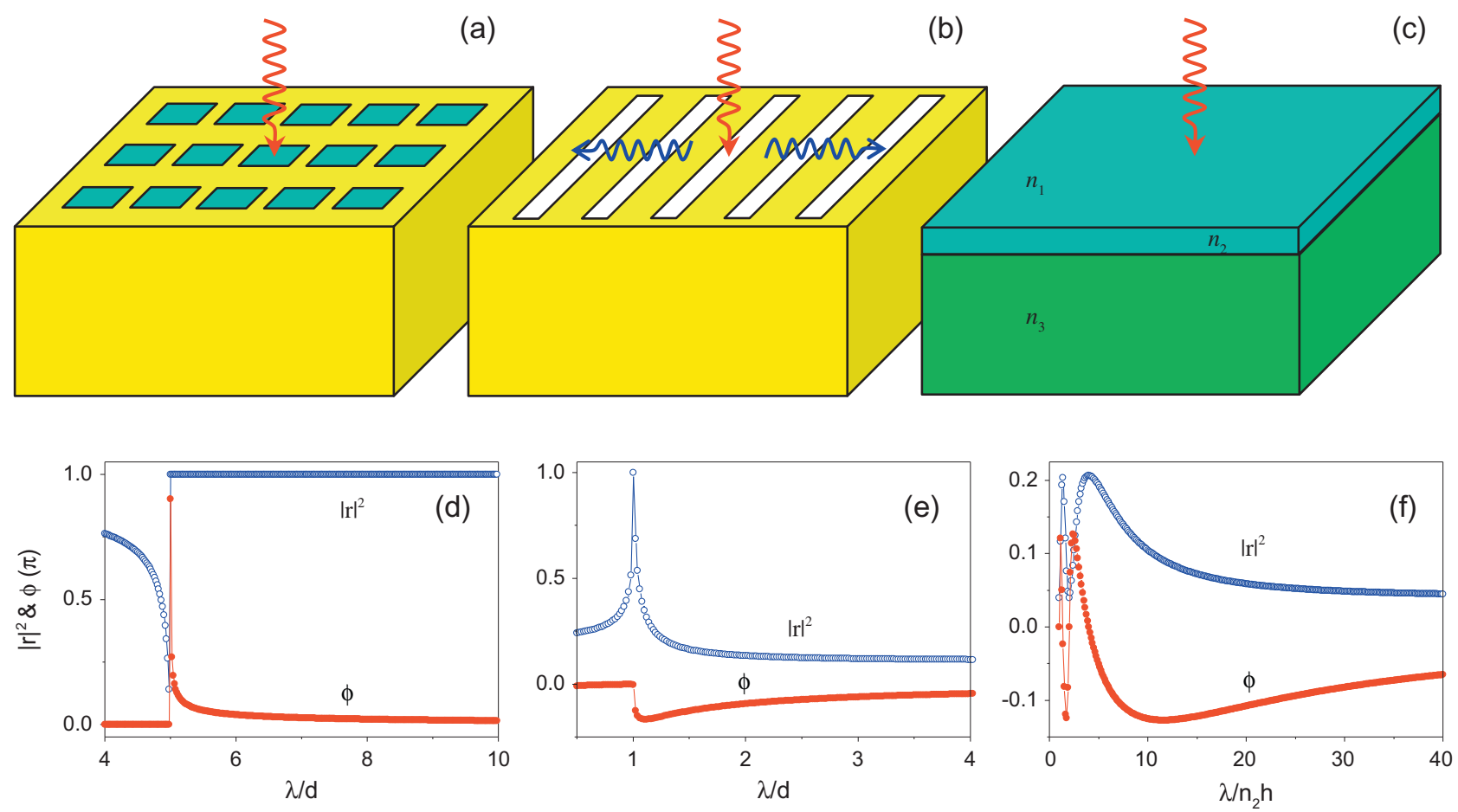

Figure 1. Reflection of light at various interfaces: (a) A semi-infinite PEC metal milled with subwavelength square holes behaves like an effective medium $(\lambda \gg d)$ with a resonant impedance (the hole width is $a$, the period is $d$, and the permittivity of hole filling medium is set as $\varepsilon_{h}=25$ ); (b) A semi-infinite PEC metal milled with periodic slits (the slit width is $a$ and the period is $d$ ) can induce the diffracted evanescent waves at the interface $(\lambda \sim d)$; (c) Light reflection by a dielectric slab (with a thickness $h$ ) sandwiched between two semi-infinite media. The reflection efficiency and phase shift for the case (a)-(c) are shown in (d)-(f) respectively. We set in (d) and (e) $a=d / 2$ and in (f), $n_{1}=1$, $n_{2}=2$, and $n_{3}=1.5$.

response [23, 51, 52]. In the effective-medium model, the impedance is then modulated depending on the permittivity which can be positive, negative, or zero in the specific frequency range. The negative (or complex) permeability or permittivity due to the resonance (or absorption) gives complex impedance and thus changes the phase shift [41].

Interestingly, an array of subwavelength holes (the complementary structure of the particles) in a semi-infinite thick and perfect-electric conducting (PEC) metal, as shown in Figure 1a, may act as an effective medium with high impedance. Following Pendry et al. [53, 54], the perforated-metal medium has an effective permeability $\mu_{\text {eff }}=8 a^{2} / \pi^{2} d^{2}$ and permittivity $\varepsilon_{\mathrm{eff}}=\left(\pi^{2} d^{2} / 8 a^{2}\right)\left(\varepsilon_{h}-\pi^{2} c^{2} / a^{2} \omega^{2}\right)$ in the long-wavelength approximation. Here, $a$ is the width of the square hole, $d$ is the lattice constant, $c$ is the light speed, and $\varepsilon_{h}\left(\varepsilon_{h} \gg 1\right)$ is the permittivity of hole filling medium. The effective permittivity is of the Drude-model form with a "plasma" frequency of the waveguide cutoff frequency. The effective impedance $Z=\left(8 a^{2} / \pi^{2} d^{2}\right) / \sqrt{\varepsilon_{h}-\pi^{2} c^{2} / a^{2} \omega^{2}}$ shows a resonance at the hole cutoff. Below the cutoff frequency, the impedance is imaginary and causes a phase shift. The effect can also be understood in terms of the evanescent-wave effect, as indicated below. Figure 1d shows an example for this case.

The device is bulky using a bulk medium as the reflectors. In the long-wavelength limit, a flat surface textured with periodic metallic features can be treated as an effective medium, which may present unusual effective surface impedance [43]. Here, the surface impedance relates the tangential electric field, including both the incoming and reflecting components, to the magnetic field at a boundary, thus determining the reflection of light directly. For example, the mushroom-like structure can be modeled as an equivalent resonant $\mathrm{LC}$ circuit, which has a surface impedance of $Z_{s}=i \omega L /\left(\omega^{2} L C-1\right)$ [43]. This impedance is inductive in the low frequencies, capacitive in the high frequencies, and exhibits a larger value at the LC resonance. Accordingly, the reflection phase of the surface decreases continuously with the frequency from $\pi$ to $-\pi$. Such a structure may also be treated as an effective medium with a resonant permeability [55].

\subsection{Phase shift due to evanescent waves}

For transverse-magnetically (TM) polarized incident light, the magnetic Fresnel's coefficient of reflection between the dielectric medium 1 and 2 (the permittivity is $\varepsilon_{1}$ and $\varepsilon_{2}$, respectively; the permeability is $\mu_{1}=\mu_{2}=1$ ) reads [56].

$$
r=\frac{1-\varepsilon_{1} k_{2}^{\perp} / \varepsilon_{2} k_{1}^{\perp}}{1+\varepsilon_{1} k_{2}^{\perp} / \varepsilon_{2} k_{1}^{\perp}} .
$$

Here, $k_{j=1,2}^{\perp}=\sqrt{k_{0}^{2} \varepsilon_{j}-k_{/ /}^{2}}$ represents the wavevector component along the normal of interface, $k_{/ /}$is the parallel wavevector component along the boundary, and $k_{0}$ is the wavevector in free space. 
Generally, the reflecting wave presents a trivial phase shift 0 or $\pi$. When the normal wavevector $k_{2}^{\perp}$ is imaginary, however, the reflection phase will deviate from 0 or $\pi$. This case can be encountered in the total reflection when the incident angle is larger than a critical value. Alternatively, the normal wavevector is also imaginary when the metal with a negative permittivity constitutes one of the interface media. The imaginary wavevector means that the wave is evanescent and decays exponentially from the interface. Accordingly, a phase shift proportional to the decay length, $\Delta \varphi \sim 2 k_{1}^{\perp} \delta=2 k_{1}^{\perp} / \operatorname{Im}\left(k_{2}^{\perp}\right)$, will be resulted. Hence, the evanescent wave will play a crucial role in tailoring the reflection phase at the boundaries although the evanescent wave cannot carry the energy away from the interface. When the transmitted wave is propagating or has a real momentum in the normal direction, the reflection phase will be 0 or $\pi$; when the transmitted wave is evanescent or has an imaginary normal momentum, the reflection phase is then changed.

The evanescent wave can also present in many other systems, such as the near field of a subwavelength nanostructure, the surface of a periodic structure, etc. [57-59]. For light incidence upon a metal surface perforated with the periodic subwavelength apertures, the propagating and evanescent waves can be excited simultaneously and the reflection phase may be changed depending on the weight of the evanescent components. We take a PEC and semi-infinite thick metal, which is milled with periodic subwavelength slits, as an example (see Figure 1b; the slit depth is also semi-infinite). By expanding the fields on the incident side as $H_{z}^{i}=e^{i k_{0} y}+\sum_{m} r_{m} e^{i\left(G_{m} x-\sqrt{k_{0}^{2}-G_{m}^{2} y}\right)}$ and that in the slits as $H_{z}^{s}=t_{0} e^{i k_{0} n_{s} y}$, the reflection coefficient for the TM polarization can be obtained with the boundary conditions as:

$$
r_{0}=1-\frac{2}{\frac{n_{s} d}{a}+\sum_{m} \frac{k_{0} g_{m}^{2}}{\sqrt{k_{0}^{2}-G_{m}^{2}}} .}
$$

Here, $G_{m}=2 \pi m / d, g_{m}=\sin \mathrm{c}(m \pi a / d), d$ is the lattice period, and $a$ is the slit width. Note that the evanescent waves on the incident side are marked by the diffraction orders with $G_{m} \geq k_{0}$ and that the evanescent effect on the transmission side can be accounted for by using a complex filling-medium index $n_{s}=n^{\prime}+i n^{\prime \prime}$

Equation (3) suggests that the reflection phase at the periodic interface is still dominated by the propagating or evanescent characteristics of the waves. If we neglect the evanescent components on both the incident and transmission sides, that is, $n_{s}$ is real and $G_{m}<k_{0}$, the reflection coefficient will be real, indicating a common phase shift of 0 or $\pi$. However, the presence of evanescent effect on either side may contribute to a deviation of phase shift. For example, Figure 1e presents the calculation results for $n_{s}=1$ and $a / d=0.5$, where a non-zero phase shift (due to the evanescent effect on the incident side) can be seen clearly.

In the long-wavelength region, the semi-infinite structure may be approximated by a homogeneous medium [54], which has an effective permittivity $\varepsilon_{x}=n_{s}^{2} d / a$ and a permeability $\mu_{z}=a / d$. Consequently, the impedance of the effective medium is $Z=a / n_{s} d$ and the reflection coefficient becomes $r=\left(n_{s} d-a\right) /\left(n_{s} d+a\right)$. This result can be recovered with the equation (3) by neglecting the evanescent terms. Thus, the phase variation due to the diffracted evanescent effect will be excluded in the simplified model. This also implies that one should be careful to use the effective-medium model when dealing with the reflection phase at the boundaries.

\subsection{Phase shift due to resonant cavities}

The reflection phase at the boundary of a slab can also be induced, which is correlated with the oscillation of waves in the slab. Consider a dielectric slab of the permittivity $\varepsilon_{2}$ and thickness $h$ sandwiched between two semi-infinite medium $1\left(\varepsilon_{1}\right)$ and $3\left(\varepsilon_{2}\right)$ (see Figure 1c). The TM-polarized light is incident with the incident angle $\theta$ upon the slab and transmits from the medium 1 to medium 3 . The reflection coefficient of the slab can be expressed as follows [56],

$$
r=\frac{r_{12}+r_{23} \exp \left(2 i k_{2}^{\perp} h\right)}{1+r_{12} r_{23} \exp \left(2 i k_{2}^{\perp} h\right)} .
$$

Here, $r_{12}$ (or $r_{23}$ ), which is determined according to the form of equation (2), is the Fresnel's reflection coefficient between the medium $\varepsilon_{1}$ and $\varepsilon_{2}$ (or $\varepsilon_{2}$ and $\varepsilon_{3}$ ); $k_{2}^{\perp}$ is the normal wavevector component in the slab.

Equation (4) shows that the reflection phase at the boundary is modulated by the dielectric slab-cavity effect. The phase shift is generally not 0 or $\pi$ even though there is no evanescent mode in the structure. To see this point more clearly, we consider the case that $n_{1}=1, n_{2}=2, n_{3}=1.5$, and $\theta=0$. The reflection efficiency and phase shift as a function of wavelength (normalized by the optical path of slab) are shown in Figure if by the open and solid circles, respectively. It can be seen that the phase shift is modified relying on the wavelength or the slab thickness $h$. Note that, when $h$ is much smaller than the wavelength, the effect is obvious as well. However, at the slab-cavity resonance where $2 k_{2}^{\perp} h=m \pi$ or $\lambda / n_{2} h=4 / m$, the reflection phase will go back to zero.

Similar cavity-dependent phase shift at the boundaries can also be present in systems such as a metal film (of finite thickness) perforated with the subwavelength slits or holes [34, 35], a thick metal milled with the periodic grooves or holes of finite depth [54, 60, 61], etc. In the case of holes, the wavelength should be smaller than the cutoff wavelength. In these cases, the evanescent modes due to the periodic textures can also generate a contribution to the phase shift which is included in the Fresnel's coefficient $r_{i j}$. This evanescent-mode effect will mix with the slab-cavity effect to induce a total reflection phase at the boundaries.

\section{SFP resonators based on metallic slits}

With the modulated reflection phase, the FP cavity length could be reduced. However, for a close-ended FP cavity defined by two physical reflectors, the lateral size of cavity is usually much larger than the wavelength. It is interesting to find that 


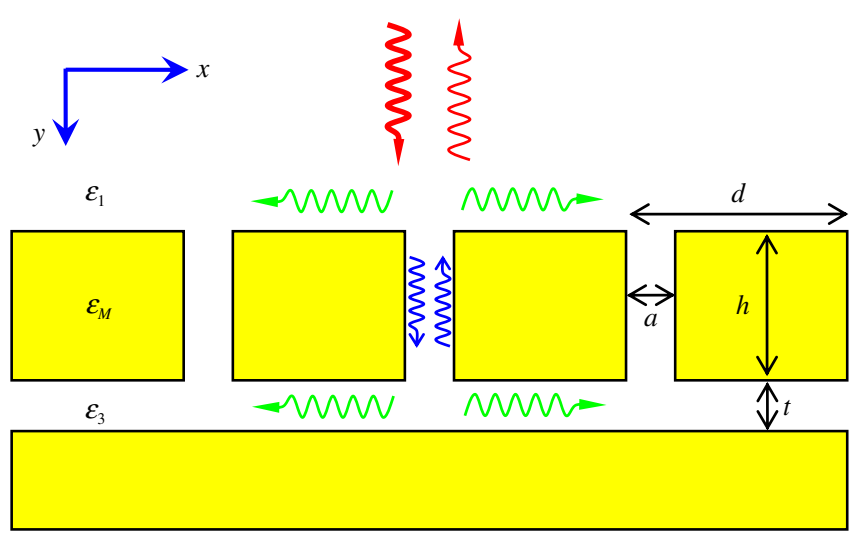

Figure 2. Schematic view and physical parameters of the plasmonic system under study. The slit grating (with a narrow slit width) and ground plane are separated by a thin dielectric spacer. The vertical narrow slits support a deep subwavelength FP-like resonance. Such resonance can be excited by a TM-polarized incident light.

single slits or slit array cut into a metal film can also behave like the common FP cavities [34, 35, 38, 62, 63], as long as the slit length is large enough. The effect originates from the fact that the slit mode couples with the surface charges on the metal walls and two effective reflection interfaces can be introduced because of the discontinuities at the slit openings. Thus, the metallic slits may act as the open-ended FP cavities which have no physical reflectors. Moreover, the width of slits can be squeezed to a deep subwavelength scale.

Compared with the FP resonance with perfect reflecting boundaries, the FP-like resonance in the open-ended slit cavities only shows a slight wavelength shift $[34,38]$. To realize the deep subwavelength effect, a modified sandwiched plasmonic structure can be used (see Figure 2). The sandwiched structure is usually composed of a thin metallic pattern (e.g., a periodic array of well-separated disks, patches, or stripes) and a back ground plane, separated by a dielectric spacer [64-73]. In such structures, horizontal or vertical resonances in the dielectric spacer can be excited, leading to enhanced electromagnetic fields and absorption. For the slit grating with a narrow slit width and a larger thickness, however, the vertical metallic slits may behave as the FP-like cavities [49, 74, 75].

According to the Section 2, the upper periodic interface provides the diffracted evanescent waves in this composite cavity structure, which modify the reflection phase at the top slitcavity openings. The dielectric spacer sandwiched between the metal grating and the ground plane acts as a thin slab cavity, which incorporates both the cavity and evanescent effects. Thus, the reflection phase at the bottom slit-cavity openings can also be modulated.

To determine the reflection phase of slit mode at the slit openings, one can write the fundamental slit mode in the slits and determine the reflection coefficients with the boundary conditions. For very narrow slits, the fundamental slit mode, consisting of downward and upward components, can be expressed as $H_{z}^{s}=A e^{i q_{0} y}+B e^{-i q_{0} y}$, where $q_{0}=k_{0} n_{h}$ is the propagation constant and $n_{h}=\sqrt{1+2 \mathrm{i} / k_{0} a \varepsilon_{M}^{1 / 2}}$ is the effective index $\left(\varepsilon_{M}\right.$ is the permittivity of the metal) [50].
By expanding the fields on the upper side as $H_{z}^{i}=\sum_{m} R_{m} e^{i k_{0}\left(\gamma_{m} x-u_{m} y\right)}$ and using the boundary conditions, we have

$$
\begin{gathered}
A+B=\sum_{m} g_{m} R_{m}, \\
R_{m}=\frac{w \varepsilon_{1} g_{m}}{u_{m}+\varepsilon_{1} \varepsilon_{M}^{-1 / 2}}(B-A) .
\end{gathered}
$$

Here, $w=n_{h} a / d, g_{m}=\sin c\left(\frac{k_{0} \gamma_{m} a}{2}\right), u_{m}=\sqrt{\varepsilon_{1}-\gamma_{m}^{2}}$, and $\gamma_{m}=m \lambda / d$. Consequently, the slit-mode electric-field reflection coefficient at the upper slit opening $(y=0), r_{1} \equiv-A / B$, can be deduced as:

$$
r_{1}=\frac{1-w \varepsilon_{1} \sum_{m} g_{m}^{2} /\left(u_{m}+\varepsilon_{1} \varepsilon_{M}^{-1 / 2}\right)}{1+w \varepsilon_{1} \sum_{m} g_{m}^{2} /\left(u_{m}+\varepsilon_{1} \varepsilon_{M}^{-1 / 2}\right)} .
$$

Equation (6) confirms that the phase shift at the upper slit openings is associated with the evanescent effect.

Similarly, by expanding the fields in the dielectric spacer and using the boundary conditions [50], one obtain

$$
\begin{gathered}
A e^{i q_{0} h}+B e^{-i q_{0} h}=\sum_{m}\left(1-\rho_{m} e^{2 i k_{0} v_{m} t}\right) g_{m} T_{m}, \\
T_{m}=\frac{w \varepsilon_{3} g_{m}\left(A e^{i q_{0} h}-B e^{-i q_{0} h}\right)}{\left(v_{m}+\varepsilon_{3} \varepsilon_{M}^{-1 / 2}\right)\left(1-\rho_{m}^{2} e^{2 i k_{0} v_{m} t}\right)},
\end{gathered}
$$

where $\quad \rho_{m}=\left(\varepsilon_{3} \varepsilon_{M}^{-1 / 2}-v_{m}\right) /\left(\varepsilon_{3} \varepsilon_{M}^{-1 / 2}+v_{m}\right), \quad$ and $\quad v_{m}=$ $\sqrt{\varepsilon_{3}-\gamma_{m}^{2}}$. Accordingly, the slit- mode electric-field reflection coefficient at the lower slit opening $(y=h), \quad r_{3} \equiv$ $B e^{-\mathrm{iq}_{0} h} /\left(-A e^{\mathrm{i} \mathrm{q}_{0} h}\right)$, can be derived as

$$
r_{3}=\frac{1-w \varepsilon_{3} \sum_{m} \mu_{m} g_{m}^{2} /\left(v_{m}+\varepsilon_{3} \varepsilon_{M}^{-1 / 2}\right)}{1+w \varepsilon_{3} \sum_{m} \mu_{m} g_{m}^{2} /\left(v_{m}+\varepsilon_{3} \varepsilon_{M}^{-1 / 2}\right)},
$$

where $\mu_{m}=\left(1-\rho_{m} e^{2 i k_{0} v_{m} t}\right) /\left(1-\rho_{m}^{2} e^{2 i k_{0} v_{m} t}\right)$ is related to the spacer thickness. Thus, besides the evanescent effect, the phase shift at the bottom slit openings also benefits from a cavity effect of the spacer. When the $m$ th-order diffraction mode is propagating, $v_{m}$ is real and thus $\mu_{m}$ is complex, giving rise to a phase contribution due to a pure cavity effect. In addition, when the diffraction mode is evanescent, a phase contribution due to the evanescent effect will emerge (here $v_{m}$ is imaginary, $\rho_{m} \approx-1$, and $\mu_{m}$ is real). In the latter case, the phase contribution can also be modulated by the spacer cavity (dependent on $t$ ).

The SFP resonance condition can thus be written as $1-r_{1} r_{3} e^{2 i q_{0} h}=0$ or $\varphi_{1}+\varphi_{3}+2 q_{0} h=2 \pi m$, where $\varphi_{1}$ and $\varphi_{3}$ is the phase shift of the slit mode at the top and bottom slit openings, respectively. Such a SFP resonance can be excited efficiently by a TM-polarized light incident from the upper side. At the SFP resonance, the electromagnetic fields will be trapped in the slit cavities and the reflection of light is greatly suppressed. Moreover, the SFP resonance can squeeze the electromagnetic energy into the spacer, giving rise to enhanced 


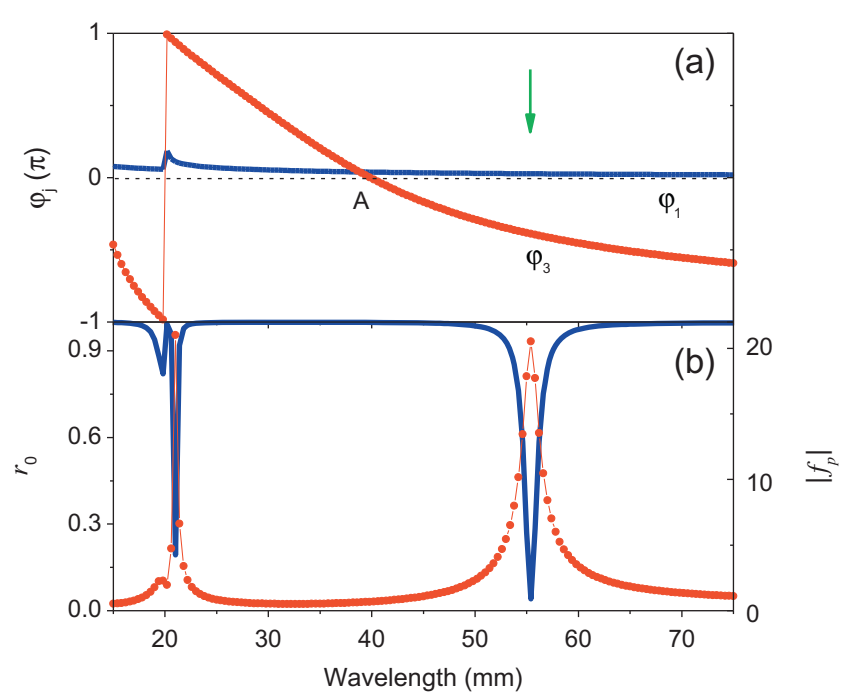

Figure 3. The SFP resonance in the microwave band for the structure with $d=20 \mathrm{~mm}, a=0.3 \mathrm{~mm}, h=5 \mathrm{~mm}$, and $t=0.1 \mathrm{~mm}$. (a) Phase shift of slit mode at the slit openings (the arrow indicates the SFP resonance); (b) Zero-order reflection (the line) and the absolute value of the FP-like resonance factor (the circles). The resonance around $20 \mathrm{~mm}$ is dominated by the grating surface modes.

radiation pressure in the system. These effects may find interesting potential applications in future. In the following, the SFP resonance and the resulted physical effects in the microwave band, $\mathrm{THz}$ and infrared range will be discussed respectively. For simplicity, throughout the rest of article, the metal is assumed to be freestanding $\left(\varepsilon_{1}=\varepsilon_{3}=1\right)$, and the metal permittivity is modeled with the Drude dispersion $\varepsilon_{M}=1-\omega_{p}^{2} / \omega(\omega+i \gamma)$, where $\omega_{p}=1.37 \times 10^{16} \mathrm{rad} / \mathrm{s}$ and $\gamma=4.05 \times 10^{13} \mathrm{rad} / \mathrm{s}$.

\section{SFP resonance in the microwave band}

To illustrate the SFP resonance in the microwave band, we use the following structural parameters: $d=20 \mathrm{~mm}$, $a=0.3 \mathrm{~mm}, h=5 \mathrm{~mm}$, and $t=0.1 \mathrm{~mm}$. Figure $3 \mathrm{a}$ presents the reflection phases of the slit mode at the cavity openings, which are dispersive in the studied spectral range: the reflection phase shift at the top $\left(\varphi_{1}\right)$ is positive but small while that at the bottom $\left(\varphi_{3}\right)$ goes from positive to negative values by crossing a zero-phase point $A$. The negative phase shift enables a zeroorder FP resonance with a long wavelength $\lambda=-4 \pi h /$ $\left(\varphi_{1}+\varphi_{3}\right)$. To confirm this point, the zero-order reflection spectrum of the whole structure at normal incidence was calculated by the equation $[50]$ :

$$
r_{0}=\left|\kappa-\frac{\eta\left(1+r_{3} e^{2 i q_{0} h}\right)}{\left(1+\theta_{1}\right)\left(1-r_{1} r_{3} e^{2 i q_{0} h}\right)}\right|^{2} .
$$

The result is shown in Figure 3b by the solid line, which exhibits a significant reflection dip at the wavelength $55.4 \mathrm{~mm}(h / \lambda=9 \%)$. This reflection dip coincides with the peak of the FP-like resonance factor $\left|f_{p}(\lambda)\right|$ (see the circles),
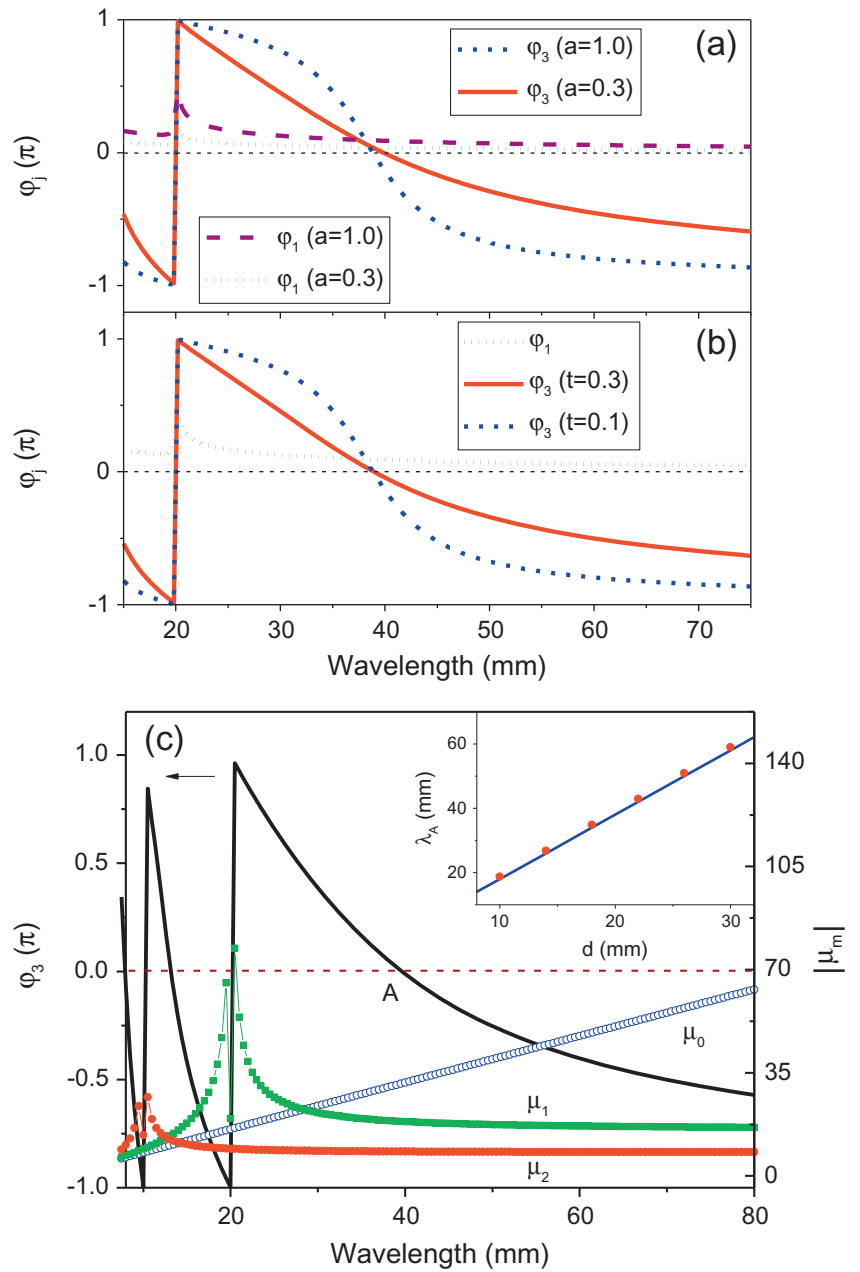

Figure 4. Dependence of phase shift on the wavelength for different slit width $a$ and spacer thickness $t$ : (a) $t=0.1 \mathrm{~mm}$, and $a=1.0$ or $0.3 \mathrm{~mm}$; (b) $a=1.0 \mathrm{~mm}$, and $t=0.1$ or $0.3 \mathrm{~mm}$. Here $d=20 \mathrm{~mm}$. The correspondence between the phase shift $\varphi_{3}$ (the line) and $\left|\mu_{m}\right|$ $(m=0,1,2)$ are shown in (c), where $d=20 \mathrm{~mm}, a=0.5 \mathrm{~mm}$, and $t=0.2 \mathrm{~mm}$. The inset of (c) shows the zero-phase point $A$ (the circles) as a function of lattice period $d$ (where we fix $a=1.0 \mathrm{~mm}$ and $t=0.2 \mathrm{~mm}$ ), which track the position of HFP mode in the spacer closely (the line).

where $f_{p}=\left(1-r_{1} r_{3} e^{2 i q_{0} h}\right)^{-1}$. Thus, a subwavelength FP resonance can be induced.

\subsection{Phase shift vs. structural sizes}

To employ the reflection phase to modulate the SFP resonance, the dependence of phase shift on the structural parameters $a$ and $t$ are investigated. In Figure 4a, two slit widths $a=1.0 \mathrm{~mm}$ and $0.3 \mathrm{~mm}$ are used and the other parameters are fixed. With the reduction of slit width, $\varphi_{1}$ decreases slightly. However, an obvious change occurs for $\varphi_{3}$ : the slope of phase curve around the zero-phase point $A$ becomes smaller (the point $A$ shows a weak redshift). This could push the SFP resonance to the longer wavelength. To understand this effect, we go back to equations (6) and (8), which show that the reflection coefficients of the slit mode are strongly dependent on the parameter 
$w$ or duty cycle $a / d$. The smaller is the duty cycle, the closer the $r_{1}$ and $r_{3}$ to unity. That means very narrow slits exhibit excellent confinement ability, as the slit mode can be reflected almost perfectly at the openings. Simultaneously, the reflection phases of slit mode also approach zero, giving rise to more flat phase curves.

The reflection phases for two spacer thickness $t=0.3 \mathrm{~mm}$ and $0.1 \mathrm{~mm}$ are shown in Figure 4b. The result is similar to that of Figure 4a, but the larger the spacer thickness, the gentler the phase variation around the point $A$ (the position of point $A$ is almost unchanged). This suggests that the spacer thickness can also be used to manipulate the reflection phase and the SFP resonance. Equation (8) suggests that this phenomenon is related to the spacer cavity effect, which is governed by $\mu_{m}$. For the small spacer thickness $(t \ll \lambda), \mu_{m}$ can be simplified as $\mu_{m} \approx v_{m} /\left(2 \varepsilon_{3} \varepsilon_{M}^{-1 / 2}-i k_{0} v_{m}^{2} t\right)$. By neglecting the term $\varepsilon_{3} \varepsilon_{M}^{-1 / 2}$ which contributes little to the phase in the microwave region, we have $\mu_{m} \approx i / k_{0} v_{m} t$ and the summation term in equation (8) becomes

$$
\sum_{m}(\ldots)=\frac{i}{k_{0} t} \sum_{m=0, \pm 1 \ldots} \frac{g_{m}^{2}}{\varepsilon_{3}-\gamma_{m}^{2}} .
$$

Equation (10) shows that each diffraction term (which is imaginary) will make a contribution to the reflection phase. Since the sum is inversely proportional to the spacer thickness $t$, the larger the $t$, the smaller the phase shift and the gentler the variation of phase curve.

To further distinguish the role of spacer-confined diffraction modes in the reflection phase, $\varphi_{3}$ and $\mu_{m}$ (here $m=0,1$, and 2) as a function of wavelength have been mapped in Figure 4c. One can see that $\left|\mu_{0}\right|$ evolves linearly with the wavelength whereas $\left|\mu_{1}\right|$ and $\left|\mu_{2}\right|$ present peaks at the phase jump around the wavelength $10 \mathrm{~mm}$ and $20 \mathrm{~mm}$. This indicates that the phase jump of $\varphi_{3}$ is related to some type of resonance mode. By checking the pole of $\mu_{m}\left(1-\rho_{m}^{2} e^{2 i k_{0} v_{m} t}=0\right)$, one obtain

$$
\frac{2 \pi m}{d}= \pm k_{0} \sqrt{\varepsilon_{3}}\left(1+\frac{\delta}{2 a}\right),
$$

where $\delta=\sqrt{2 / \mu_{0} \omega \sigma}$ is the skin depth of metal. This corresponds to the $m$ th-order "SPP resonance" in the spacer region. In the microwave band, the skin depth $(\sim \mu \mathrm{m})$ is very small compared with the slit width. Thus, the resonance wavelength, $m \lambda= \pm n_{3} d(1+\delta / 2 a)$, appears very close to the Rayleigh anomaly.

In the long-wavelength region, however, the role of zero-order diffraction mode becomes significant. We note from equation (10) that the zero-order term and the high-order terms are just of the opposite role for the reflection phase. In a particular wavelength ( $A$ point), the role of all terms may be canceled completely, giving rise to a null phase shift. This particular case satisfies the following condition:

$$
\frac{g_{0}^{2}}{\varepsilon_{3}}=\frac{2 g_{1}^{2}}{(\lambda / d)^{2}-\varepsilon_{3}}+\frac{2 g_{2}^{2}}{(2 \lambda / d)^{2}-\varepsilon_{3}}+\ldots
$$

We found that the wavelength thus determined is very close to $m \lambda \approx 2 \sqrt{\varepsilon_{3}}(d-a)$, i.e., the wavelength of horizontal FP (HFP) resonance in the spacer (beneath the metal stripes). This can be seen from the inset of Figure $4 \mathrm{c}$, where the grating period $d$ is changed ( $a$ is fixed as $0.5 \mathrm{~mm}$ ) and the theoretically obtained positions (the circles) of the point $A$ approach that of the HFP resonance (the line). We thus attribute the zero phase-shift at the point $A$ to the HFP resonance in the spacer. On the left- or right-hand side of point $A$, the role of zero-order mode will be defeated by or surpass that of high-order modes, yielding the positive or negative reflection phase.

\subsection{Reflection spectrum vs. structural sizes}

The influence of structural parameters on the SFP resonance can be further demonstrated by studying the reflection spectrum. The reflection spectrum as a function of wavelength and slit cavity length is shown in Figure 5a. With the increase of cavity length, the reflection minimum caused by the SFP resonance (the red-colored part) redshifts obviously. For the cavity length $h=1 \sim 10 \mathrm{~mm}$, the resonance wavelength varies from $42 \mathrm{~mm}$ to $64 \mathrm{~mm}$ with $h / \lambda=2.4 \% \sim 15.6 \%$. The increases of spacer thickness $t$ can also redshift the SFP resonance due to the variation of reflection phase. This point is verified in Figure $5 \mathrm{~b}$. When $t$ is changed from $0.1 \mathrm{~mm}$ to $0.7 \mathrm{~mm}$, for example, the resonance wavelength will shift from $50 \mathrm{~mm}$ to around $90 \mathrm{~mm}$. However, the reflection dip becomes less significant. In addition, we can also manipulate the SFP resonance with the slit cavity width $a$ (see Figure $5 \mathrm{c}$ ). The smaller is the cavity width, the larger the resonance wavelength. For $a=0.1 \mathrm{~mm}$ with a duty cycle as small as $0.5 \%$, the resonance wavelength is around $76 \mathrm{~mm}(h=5 \mathrm{~mm})$ and the absorption efficiency of microwave is up to $95 \%$. When normalized to the duty cycle of grating, the enhancement of absorption attains 190.

Experimentally, we constructed two samples to demonstrate such an effect. The samples are composed of an aluminum slit grating and a planar ground plane, which are separated by a tunable air gap (the grating covers an area of $300 \mathrm{~mm} \times 300 \mathrm{~mm}$ ). For both samples, the lattice period is $d=20 \mathrm{~mm}$, slit width $a=1 \mathrm{~mm}$, and the slit cavity length is $5 \mathrm{~mm}$ and $10 \mathrm{~mm}$, respectively (see Figure 6a). The TM-polarized microwave (the magnetic field is parallel to the slits) is incident with an angle of $5^{\circ}$. The reflected microwave is collected and measured with a microwave network analyzer (HP8150C). Figure 6b shows the measured (the circles) and calculated (the line) reflection spectra for the sample with $h=5 \mathrm{~mm}$ and $t=0.15 \mathrm{~mm}$. A reflection dip corresponding to the SFP resonance at $6.3 \mathrm{GHz}$ (or $47.6 \mathrm{~mm}$ ) has been observed. By tuning the air gap thickness $t$ (from $0.15 \mathrm{~mm}$ to $0.9 \mathrm{~mm}$ ), a redshift of SFP from 6.3 GHz to $3.8 \mathrm{GHz}$ can be seen (Figure $6 \mathrm{c}$ ). The spectral position of experiments agrees well with the theory. However, the measured reflection efficiency at the resonance is lower than the theoretical prediction. This can be attributed to the finite area of the samples, where the diffraction modes in the air gap can escape from the four sample ridges, thus reducing the actual reflection of microwave. In addition, for $h=10 \mathrm{~mm}$ 

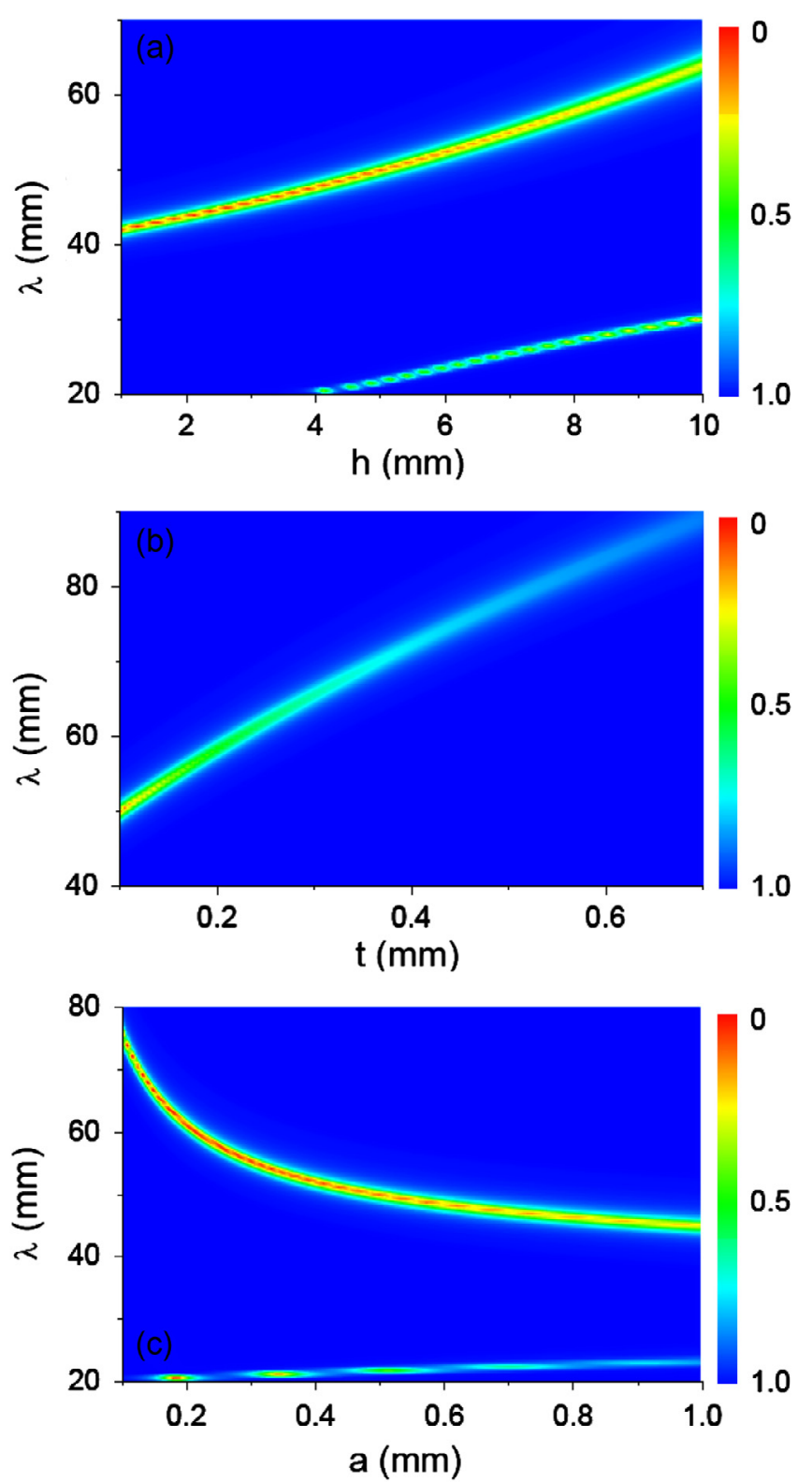

Figure 5. Reflection spectrum as a function of wavelength and structure sizes: (a) $a=0.5 \mathrm{~mm}, t=0.1 \mathrm{~mm}$; (b) $a=0.5 \mathrm{~mm}$, $h=5 \mathrm{~mm}$; and (c) $h=5 \mathrm{~mm}, t=0.1 \mathrm{~mm}$. The lattice constant is fixed as $d=20 \mathrm{~mm}$.

and $t=0.3 \mathrm{~mm}$ (see Figure 6d), two reflection dips around $4.3 \mathrm{GHz}(70 \mathrm{~mm})$ and $10.8 \mathrm{GHz}(27.8 \mathrm{~mm})$ can be found. Compared with $h=5 \mathrm{~mm}$, the low-frequency dip for $h=10 \mathrm{~mm}$ shows an obvious redshift. We also measured the microwave reflection for different spacer thickness and the spectral position as a function of $t$ is plotted in Figure 6e. A good agreement between theory and experiment is seen.

\subsection{Electromagnetic pressure vs. structural sizes}

The optical force may provide a convenient tool for manipulating small objects [76, 77]. In the cavity opto- mechanics, the optical force can provide a strong coupling between the optical mode and mechanical oscillation [78-81]. Optical forces can be enhanced employing the resonance modes in the plasmonic systems [82-88]. Here we investigate the electromagnetic pressure associated with the SFP resonance which supports an efficient confinement of electromagnetic energy in the slit cavities. The coupling between the slits and spacer also enhances the electromagnetic fields in the spacer at the SFP resonance (but not at the HFP resonance). Such field enhancement may lead to an amplified electromagnetic pressure.

The numerical simulations show that the antiparallel (horizontal) currents can be excited near the spacer, which generates a repulsive or positive force [50]. Accompanying the oscillating currents, positive and negative charges accumulate on the upper or lower side of the spacer, giving rise to an attractive or negative force. The photonic repulsive pressure, $P_{m}=(1 / 4 d) \int_{-d / 2}^{d / 2} \mu_{0}\left|H_{3}\right|^{2} \mathrm{~d} x$ (proportional to the magnetic energy density in the spacer), may be balanced by the attractive pressure $P_{e}=-(1 / 4 d) \int_{-d / 2}^{d / 2} \varepsilon_{0}\left|E_{2}\right|^{2} \mathrm{~d} x$ (proportional to the electric energy density in the spacer) [50] due to the equipartition of energy. However, the situation will be quite different considering the following two effects.

One is the field penetration effect in the metal [89], which suppresses the magnetic field/energy due to a sharing of energy by the kinetic energy of free electrons. The larger the share of the electronic kinetic energy, the smaller the magnetic energy and the weaker the repulsive force. This effect is important in the visible frequency range. In the microwave band, the electron kinetic energy $u_{K}=L_{e} i^{2} / 2$, where the kinetic inductance $L_{e} \propto 2 \mu_{0} / k_{p}^{2} \delta,\left(k_{p}=2 \pi / \lambda_{p}, \lambda_{p}\right.$ is the plasma wavelength, and $\delta$ is the skin depth) is small compared with the magnetic energy $u_{M}=L_{m} i^{2} / 2$, where the Faraday inductance $L_{m} \propto \mu_{0} t(t$ is the spacer thickness). Thus, the kinetic energy (leading to internal inductance) will can be neglected in the microwave regime $\left(L_{\mathrm{e}} / L_{\mathrm{m}} \sim \lambda_{\mathrm{p}}^{2} / 2 \pi^{2} \delta \mathrm{t}\right)$. The other effect is the field leakage effect [90], where the "leakage" of electric or magnetic energy from the spacer to the surrounding environment (especially the slit FP cavities) plays a crucial role. Hence, the subwavelength FP cavities may modulate the electromagnetic pressure strongly.

Figure 7 presents the calculated (normalized) electromagnetic pressure as a function of wavelength and structural parameters. The pressure normalized by the common value induced on a flat PEC surface can be written as [50]:

$$
\frac{P_{a}}{P_{0}}=\frac{1}{4} \sum_{m}\left(1-\gamma_{m}^{2}\right)\left|T_{m}\left(1-\rho_{m}\right) e^{i k_{0} v_{m} t}\right|^{2}
$$

Here, $T_{m}$ is the amplitude of the $m$ th-order diffraction mode in the spacer:

$$
T_{m}=\frac{g_{m}\left[\left(1+\theta_{1}\right)\left(1+\theta_{3}\right)\right]^{-1}}{\left(1+\tau_{m}\right)\left(1-\rho_{m}^{2} e^{2 i k_{0} v_{m} t}\right)} \chi f_{p} e^{i q_{0} h} .
$$

Note that $T_{m}$ is proportional to the FP-like resonance factor $f_{p}$. Figure $7 \mathrm{a}$ shows that the pressure for the long-wavelength (zero-order FP) resonance is positive whereas that for the shorter-wavelength resonance is negative. In the former case, the energy "leaked" to the FP cavities is mainly the electric 

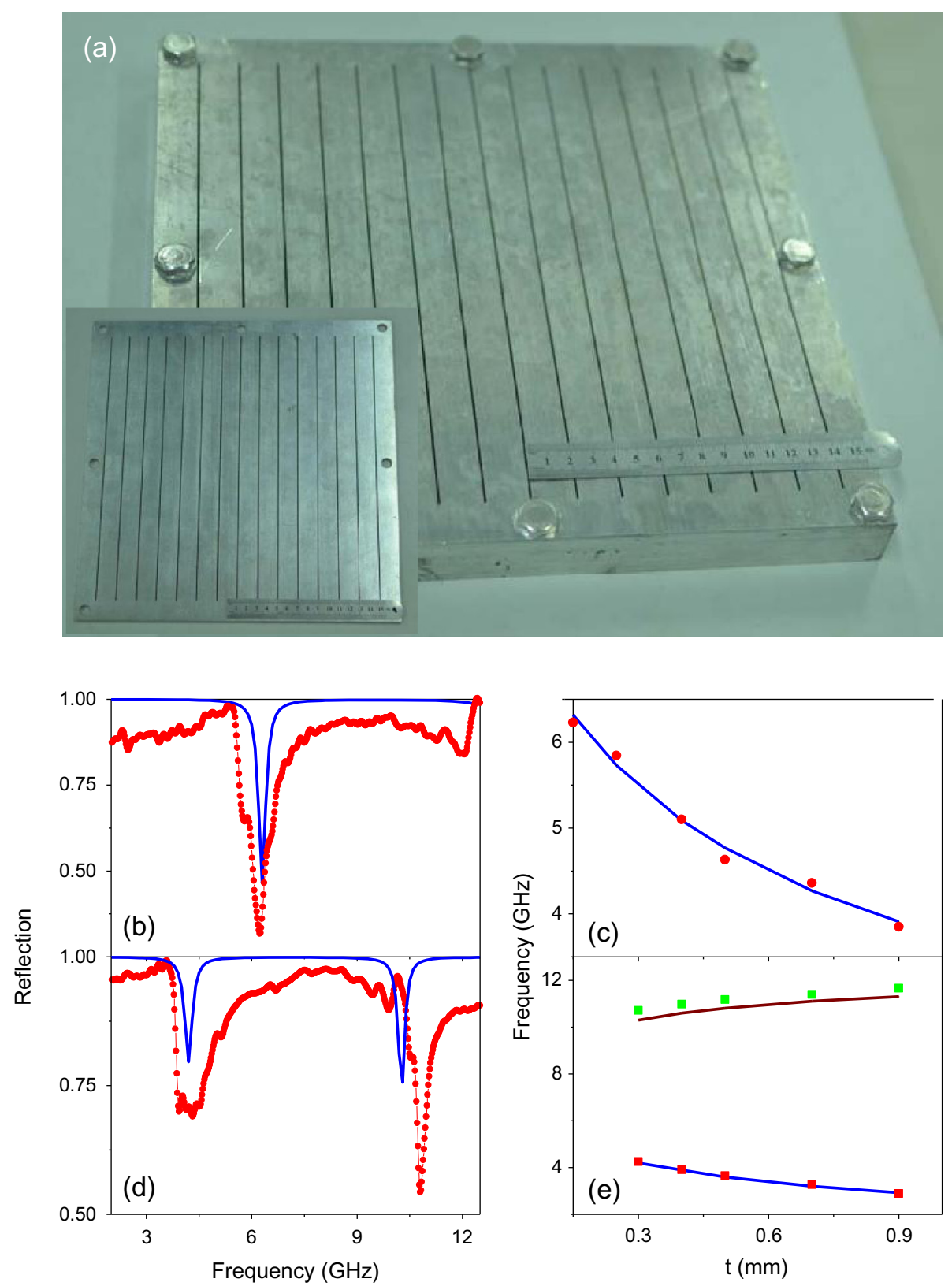

Figure 6. Experimental results for the microwave band: (a) Photographs of the fabricated samples. The lattice constant of the two samples is $d=20 \mathrm{~mm}$, the slit width is $a=1 \mathrm{~mm}$, and the grating thickness is $h=5 \mathrm{~mm}$ and $10 \mathrm{~mm}$, respectively. The measured (the circles) and calculated (the line) reflection spectra are presented in (b) for $h=5 \mathrm{~mm}, t=0.15 \mathrm{~mm}$ and (d) for $h=10 \mathrm{~mm}, t=0.3 \mathrm{~mm}$, respectively. The measured (the circles) and calculated (the lines) resonance wavelength of the two samples as a function of spacer thickness $t$ are shown in (c), $h=5 \mathrm{~mm}$, and (e), $h=10 \mathrm{~mm}$, respectively.

energy, which suppresses the attractive force. In the latter case, the antiparallel currents can also be induced in the metallic slit walls, due to the larger phase retardation in the slits; thus the energy "leaked" to the FP cavities becomes the magnetic energy, which suppresses the repulsive force. The effect becomes more significant with the increase of FP cavity length. For $h=10 \mathrm{~mm}$ and $a=0.5 \mathrm{~mm}$, the normalized pressure attains $\sim 1000$ at the wavelength $64 \mathrm{~mm}$ and -500 at $30 \mathrm{~mm}$.

The positive and negative pressure will decrease with the spacer thickness $t$ (see Figure 7b), as the increase of $t$ may reduce the electromagnetic energy density in the spacer. This may be useful for the dynamic control of the force. In addition, a reduction of slit width $a$ can also boost the electromagnetic pressure, especially that for the zero-order FP resonance (see Figure 7c). For example, when $a$ is reduced from $1 \mathrm{~mm}$ to $0.1 \mathrm{~mm}$, the normalized pressure changes from less than 500 (at $58 \mathrm{~mm}$ ) to around 2000 (at $100 \mathrm{~mm}$ ). With the decrease of slit width, the coupling between the adjacent metal stripes becomes stronger. Thus the currents at the bottoms of metal stripes may induce the horizontal electric dipoles crossing the narrow slits. Accordingly, the original (vertical) electric dipoles developed crossing the spacer are suppressed, which leads to a 

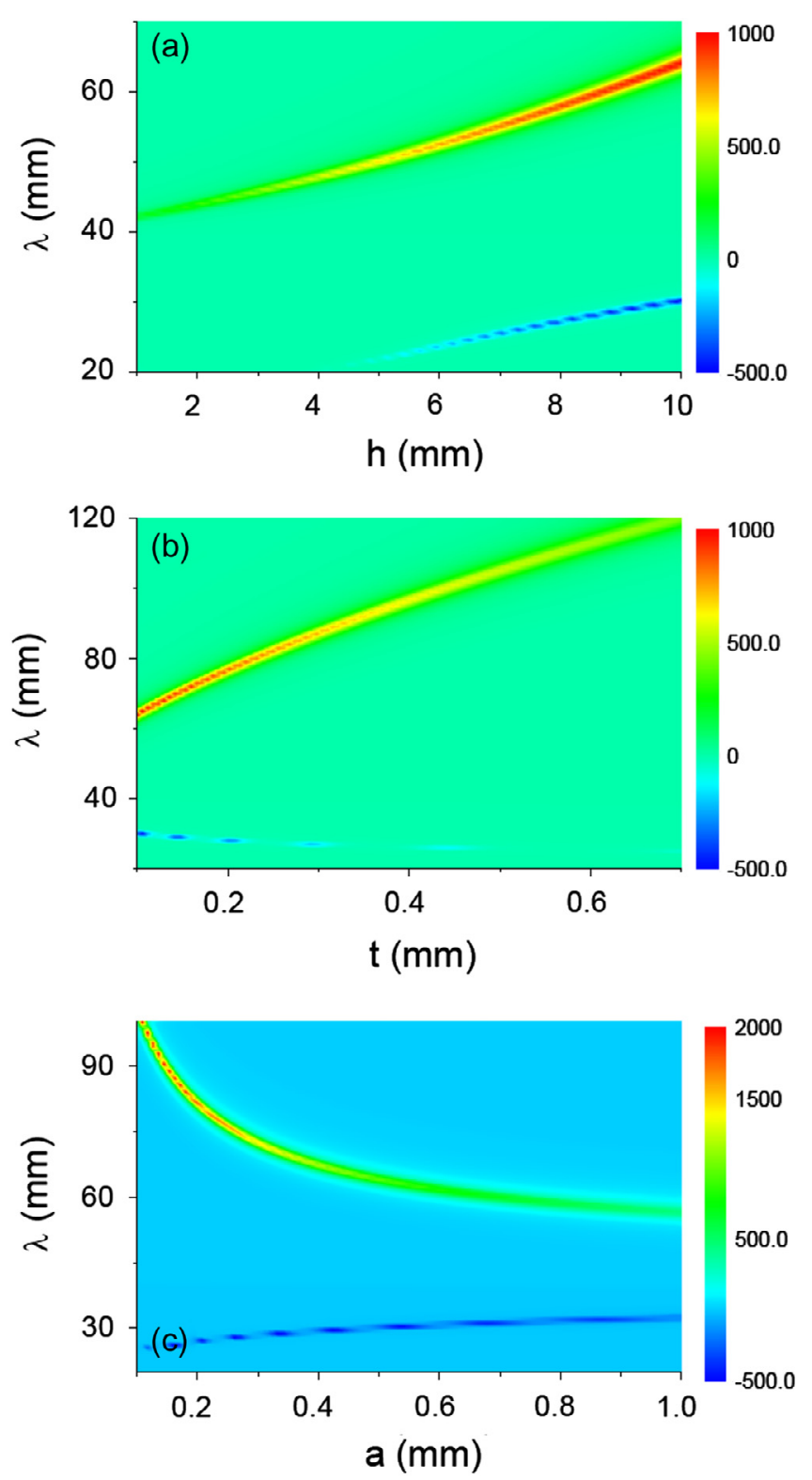

Figure 7. The SFP-resonance induced electromagnetic pressure in the microwave band as a function of wavelength and structure sizes: (a) $a=0.5 \mathrm{~mm}, t=0.1 \mathrm{~mm}$; (b) $a=0.5 \mathrm{~mm}, h=10 \mathrm{~mm}$; and (c) $h=10 \mathrm{~mm}, t=0.1 \mathrm{~mm}$. The lattice constant is fixed as $d=20 \mathrm{~mm}$.

decrease of attractive force and enhancement of the total electromagnetic pressure.

\section{SFP resonances at high frequencies}

The SFP resonance effect may be extended to higher frequencies such as the $\mathrm{THz}$ or infrared regime by scaling the structural sizes with the wavelength $[49,91]$. The difference is that the permittivity of metal becomes significantly smaller than that in the microwave band. Consequently, the skin depth scaled by the wavelength, $\delta / \lambda=\left[2 \pi \operatorname{Im}\left(\varepsilon_{M}^{1 / 2}\right)\right]^{-1}$, will be increased. This will modify the SFP resonance effect and the electromagnetic pressure. In the following, the $\mathrm{THz}$ and infrared region will be considered using two examples.

\subsection{Phase shift at the cavity openings}

The reflection phases at the cavity openings are checked again, considering the variation of slit width $a$ and spacer thickness $t$. The result for varying the slit width $a$ is similar to that obtained in the microwave band (see Figure 4a), but the case will be somewhat different concerning the variation of $t$. Figure $8 \mathrm{a}$ shows the wavelength dependence of reflection phase at the THz band for two values of $t=0.1 \mu \mathrm{m}$ and $0.5 \mu \mathrm{m}$ $(d=20 \mu \mathrm{m}$ and $a=0.5 \mu \mathrm{m})$. We see that, besides the slope of phase curve, the position of zero-phase point $A$ varies with $t$ (in the microwave band, the point $A$ is almost independent of $t$ ). To see the effect more clearly, the inset of Figure 8a plots the position of $A$ as a function of spacer thickness $t$ (the circles). For the smaller $t(t<0.3 \mu \mathrm{m})$, a redshift with the decrease of $t$ is seen. However, for the larger $t(t>0.3 \mu \mathrm{m})$, an opposite result can be obtained. As a comparison, Figure $8 \mathrm{~b}$ also shows the wavelength dependence of reflection phase at the infrared band for two values of $t=10 \mathrm{~nm}$ and $30 \mathrm{~nm}(d=2000 \mathrm{~nm}$ and $a=50 \mathrm{~nm}$ ). The dependence of point $A$ on the spacer thickness $t$ is more significant, as shown by the circles in inset of Figure 8b. Thus, both the position of $A$ and the slope of phase curve are governed by the spacer thickness $t$, which may in turn modulate the reflection phase $\varphi_{3}$.

To highlight the spacer-cavity effect in the high frequencies, $\varphi_{3}$ and $\mu_{m}$ (here $m=0,1$, and 2) as a function of wavelength are also calculated. Here we only take the infrared regime as an example and the results are shown in Figure 8c. For the infrared regime and the smaller $t, \mu_{m} \approx i v_{m} / k_{0}\left(2 \varepsilon_{3} \delta_{0}+v_{m}^{2} t\right)$, where $\delta_{0}=c / \omega_{p}$ is the skin depth. Thus, $\mu_{0}$ is suppressed due to the field penetration effect; $\mu_{1}$ and $\mu_{2}$ exhibit zero at the Rayleigh anomaly $\left(v_{m}=0\right)$ and maxima at the SPP resonance $(2 \pi m /$ $d= \pm k_{0} n_{t}$, where a phase jump occurs; $n_{t}$ is the effective refractive index of air spacer). Moreover, due to the field penetration effect, the zero-phase point $A$, corresponding to $\sum_{m} g_{m}^{2} /\left(2 \varepsilon_{3} \delta_{0}+v_{m}^{2} t\right) \sim 0$, becomes dependent on $t$ significantly.

We have also fitted the position of point $A$ with a simple HFP model with $\lambda \sim 2 n_{t}(d-a)$. In the $\mathrm{THz}$ regime, $n_{t}=\sqrt{1+\left(\delta_{0} / t\right) \sqrt{2+2 \sqrt{1+\gamma^{2} / \omega^{2}}}}$; and in the infrared regime, $n_{t}=\sqrt{1+2 \delta_{0} / t}$. The predictions of the simple HFP model are indicated by the lines in inset of Figures $8 \mathrm{a}$ and $8 \mathrm{~b}$, respectively. For the smaller $t$, the circles and the line agree well with each other. For larger $t$, a slight deviation can be seen which is due to the fact that the simple HFP model neglects the field decaying length (or reflection phase) at the periodic spacer openings. This decaying length is negligible for the smaller $t$ but becomes noticeable for the larger $t$. Moreover, the agreement in the infrared regime seems better than that in the THz band, as the fields can be well confined due to the strong plasmonic effect. 

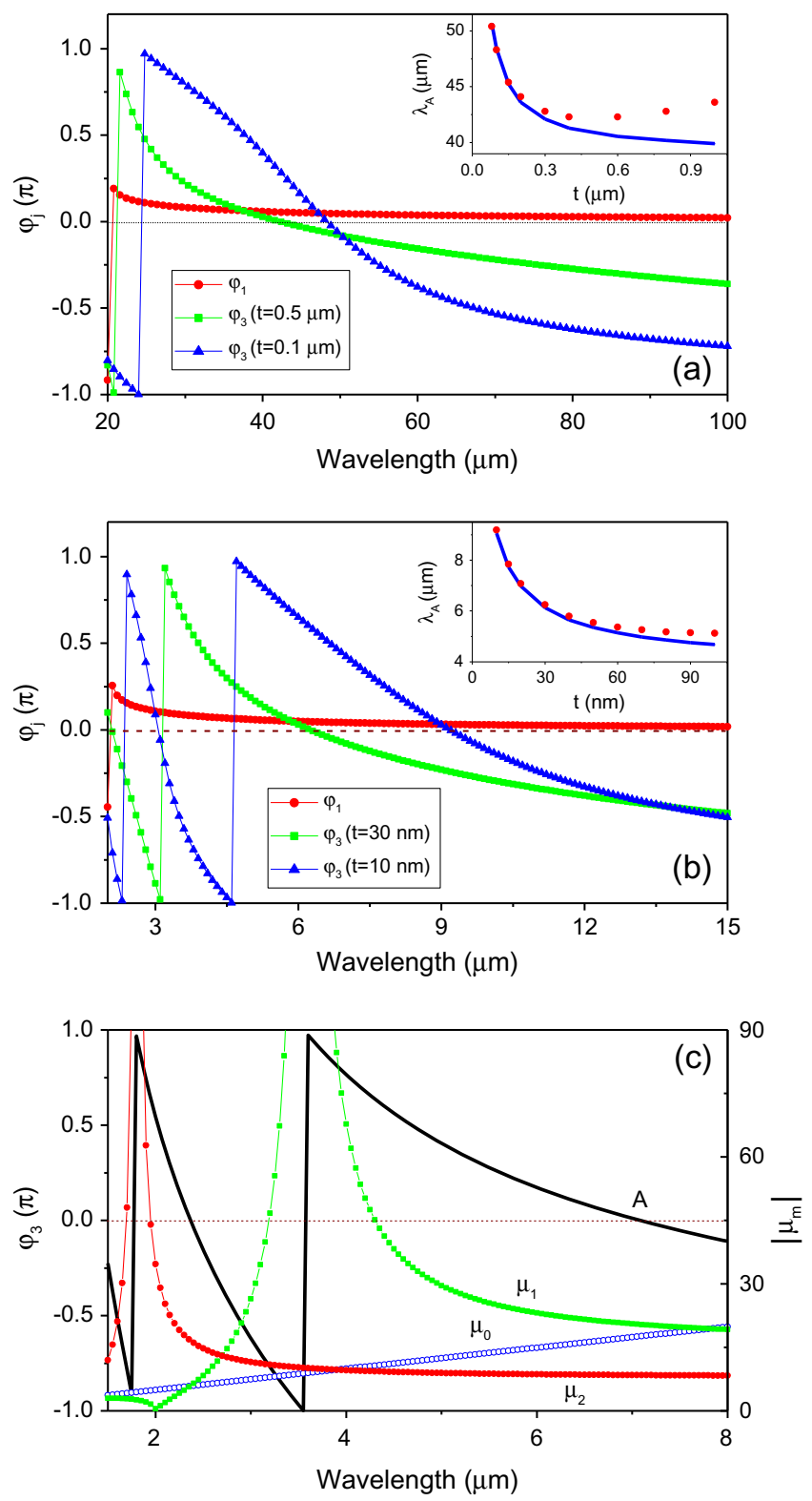

Figure 8. (a) Phase shift in the $\mathrm{THz}$ band for two spacer thickness $t=0.1$ and $0.5 \mu \mathrm{m}(d=20 \mu \mathrm{m}, a=0.5 \mu \mathrm{m})$; (b) Phase shift in the infrared region for $t=10$ and $30 \mathrm{~nm}(d=2000 \mathrm{~nm}, a=50 \mathrm{~nm})$. The inset of (a) and (b) shows, respectively, the zero-phase point $A$ as a function of $t$ for the two frequency band, where the circles represent the theoretical results and the line a simple HFP model. The correspondence (in the infrared region) between the phase shift $\varphi_{3}$ (the line) and $\left|\mu_{m}\right|(m=0,1,2)$ are shown in (c), where $d=2000 \mathrm{~nm}, a=50 \mathrm{~nm}$, and $t=20 \mathrm{~nm}$.

\subsection{Reflection spectrum}

The reflection spectra for the $\mathrm{THz}$ band as a function of wavelength and structure parameters $h, t$, and $a$ are plotted in Figures $9 \mathrm{a}-9 \mathrm{c}$, respectively. The qualitative results are very similar to that for the microwave band (see Figure 5). However, due to a relatively larger penetration length, the absorption of $\mathrm{THz}$ wave becomes more significant, yielding a wider width of absorption spectrum. In Figure 9b, the absorption increases with decreasing spacer thickness $t$; but for smaller $t$ $(t<0.2 \mu \mathrm{m})$, a degeneration of absorption can be seen instead (similar effect, not shown in Figure 5b, also exists in microwave band for very small $t$ ). According to equation (14), we have $T_{m} \propto f_{p} /[t+(\ldots)]$, where $f_{p}$ was found to reduce with a decrease of $t$. Thus, the field amplitude and absorption will be suppressed in the case of small as well as large $t$. For completeness, the corresponding results for the infrared band are shown in Figures 9d-9f. The results, except for Figure 9e, are also similar to that obtained in the THz band. In Figure 9e, with the decrease of spacer thickness $t$, the SFP resonance wavelength blueshifts when $t>40 \mathrm{~nm}$ and redshifts when $t<40 \mathrm{~nm}$. The redshift of SFP resonance at the smaller $t$ is mainly caused by the shift of the zero-phase point. This effect is more significant in the infrared region. The dependence of resonance on the spacer thickness may be used to modulate the SFP effect or measure the tiny motion with a high accuracy.

\subsection{Electromagnetic pressure}

The electromagnetic pressure at the $\mathrm{THz}$ band as a function of wavelength and structural parameters $h, t$, and $a$ are shown in Figures $10 \mathrm{a}-10 \mathrm{c}$, respectively. We found that the pressure at the $\mathrm{THz}$ band can also be enhanced at the SFP resonance. At the long-wavelength FP resonance, the pressure is positive; and at the short-wavelength resonance, the pressure is negative. To understand this effect, we should note that the kinetic energy of free electrons will play a role. At the long-wavelength SFP resonance, the electric energy is "leaked" to the slit FP cavities, thus reducing the attractive force. However, the kinetic energy of free electrons suppresses the magnetic energy and the repulsive force slightly. Thus, a total positive but smaller pressure can be induced, which increases with the cavity length. At the short-wavelength resonance, on the other hand, the magnetic energy is "leaked" due to the slit-FP cavity effect and field penetration effect (electron kinetic energy). This leads to a negative pressure.

In Figure 10b, a decrease of pressure (at long-wavelength resonance) with decreasing $t$ can be observed. For the smaller $t$, although the electric and magnetic field energy density can be enhanced, the ratio between the kinetic energy of free electrons and magnetic energy $L_{e} / L_{m} \propto \lambda_{p}^{2} / \delta t$ increases, which suppresses the total force (here $\delta=\delta_{0} \sqrt{2\left(1+\gamma^{2} / \omega^{2}\right) /\left(1+\sqrt{1+\gamma^{2} / \omega^{2}}\right)}$ is the skin depth). At the short-wavelength SFP resonance, nonetheless, the enhancement of the role of free electrons gives rise to an increase of negative pressure. However, when $t$ is large enough (not shown here), the total pressure will be reduced eventually because of the decrease of field amplitude and the attractive/ repulsive force.

The electromagnetic pressure at the infrared regime is quite different, as shown in Figure 10d-10f. The pressure associated with the SFP resonance is negative and can be enhanced by two orders of magnitude. In this regime, the kinetic energy of free electrons may exceed the magnetic energy and thus play a dominant role (suppresses the repulsive force and yields a negative pressure). In Figure 10d, with the increase of slit length and "leakage" of electric (or magnetic) energy, the pressure at the 

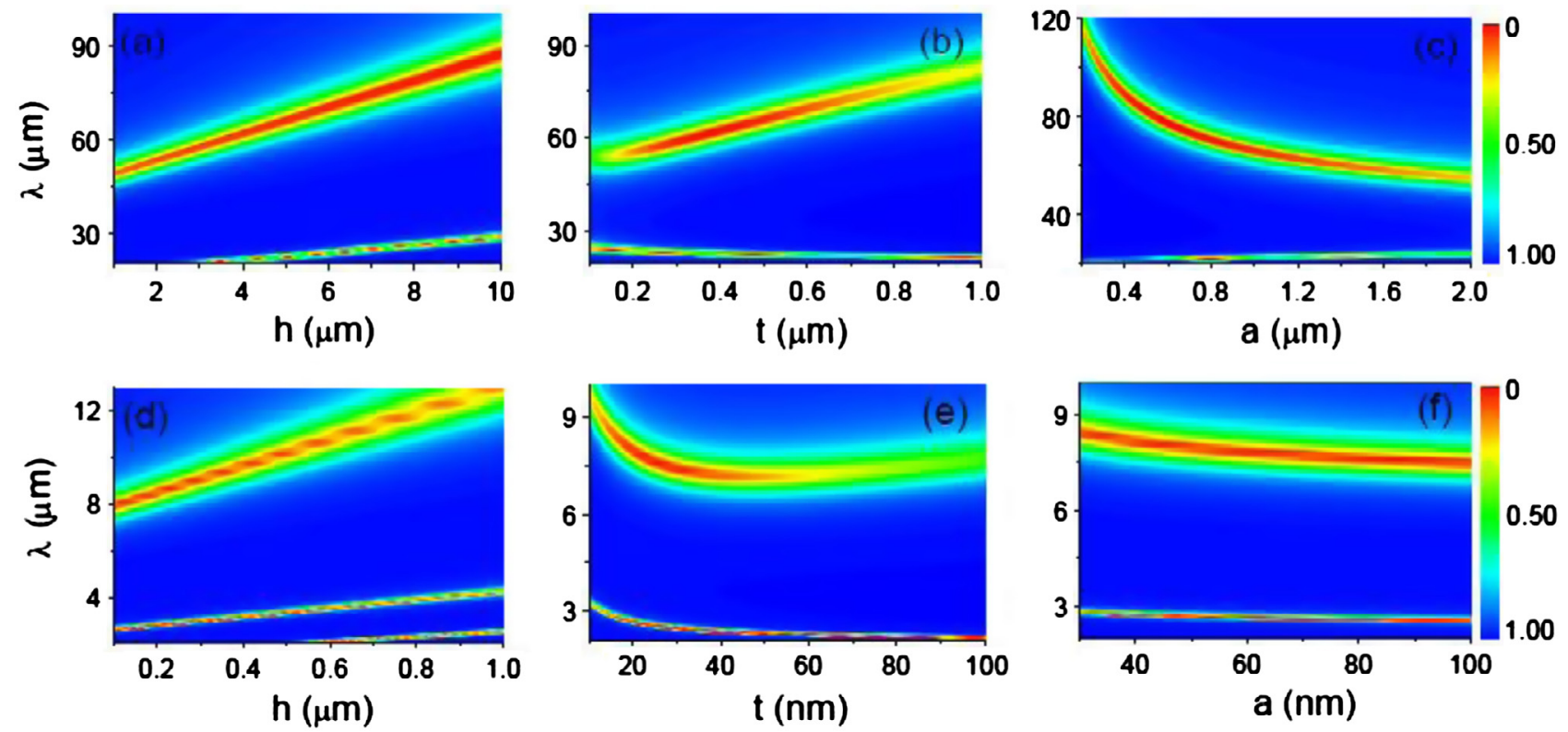

Figure 9. Reflection spectra as a function of wavelength and structure sizes for the THz $(\mathrm{a}-\mathrm{c})$ and infrared $(\mathrm{d}-\mathrm{f})$ regime. In the THz band, we fix $d=20 \mu \mathrm{m}$ and set: (a) $a=1 \mu \mathrm{m}, t=0.5 \mu \mathrm{m}$; (b) $a=1 \mu \mathrm{m}, h=5 \mu \mathrm{m}$; and (c) $h=5 \mu \mathrm{m}, t=0.5 \mu \mathrm{m}$. In the infrared regime, we fix $d=2000 \mathrm{~nm}$ and set: (d) $a=50 \mathrm{~nm}, t=20 \mathrm{~nm}$; (e) $a=50 \mathrm{~nm}, h=100 \mathrm{~nm}$; and (f) $h=100 \mathrm{~nm}, t=20 \mathrm{~nm}$.
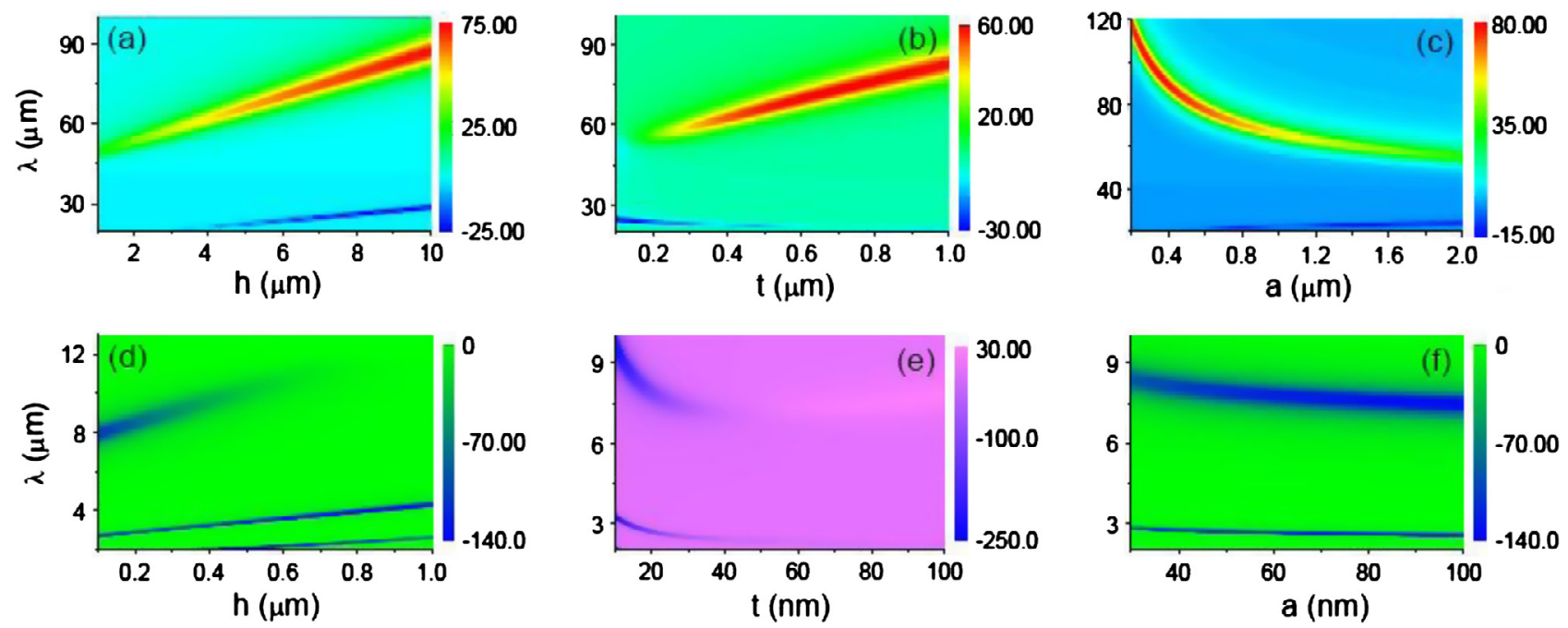

Figure 10. Enhanced electromagnetic pressure as a function of wavelength and structure sizes for the THz (a-c) and infrared (d-f) regime. The structure parameters are the same as those set in Figure 9.

long- (or short-) wavelength SFP resonance will be reduced (or enhanced). In Figure 10e, a drastic decrease of optical pressure with the spacer thickness $t$ can be found. This can be attributed mainly to the decrease of ratio between the electron kinetic energy and magnetic field energy. When $t$ is large enough, a positive pressure at the SFP resonance can even be achieved. In addition, the decrease of slit width $a$ results in a slight decrease of negative pressure at the long-wavelength resonance (see Figure 10f). This is contrary to the result in the $\mathrm{THz}$ or microwave band. However, the physical origin is common: the coupling between the adjacent metal stripes increases, leading to an enhanced "leakage" of electric energy to the slit FP cavities.

\section{Some additional discussions}

\subsection{Incident-angle dependence}

The discussion presented above has been focused on the normal incidence of incident light. What will happen then when the incident angle is increased? Taking the infrared regime as an example, we plot in Figure 11 the phase shift and reflection 

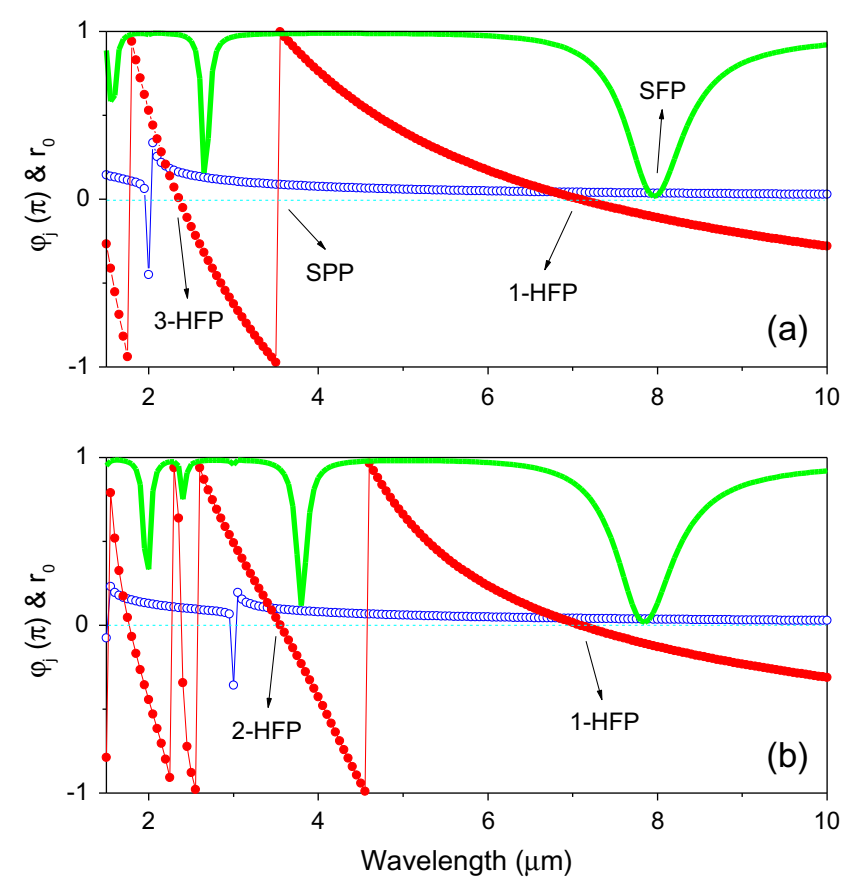

Figure 11. Incident-angle dependence of phase shift and reflection spectrum: (a) $\theta=0^{\circ}$ and (b) $\theta=30^{\circ}$. Here, $\varphi_{1}, \varphi_{3}$, and $r_{0}$ are labeled by the open circles, solid circles, and the line, respectively. As an example, the structure parameters are set as $d=2000 \mathrm{~nm}$, $a=50 \mathrm{~nm}, h=100 \mathrm{~nm}$, and $t=20 \mathrm{~nm}$.

spectrum for normal (a) and off-normal (b) incidence. For offnormal incidence, $\gamma_{m}$ in previous equations should be revised as $\gamma_{m}=\sqrt{\varepsilon_{1}} \sin \theta+m \lambda / d$. For normal incidence, the odd-order HFP resonance modes exist, which cause in the spectrum two zero-phase points and allow for the formation of two SFP resonances. For off-normal incidence, the positions of the oddorder HFP resonance modes remain unchanged, but the SPP resonance mode corresponding to the phase jump shifts to the long wavelength. This increases the slope of phase curve and leads to a slight blueshift of the SFP resonance around $8 \mu \mathrm{m}$. Moreover, when the incident angle is nonzero, the even-order HFP resonance modes (which are inactive in the normal incidence) can also be resulted due to the breaking of system symmetry. Consequently, new reflection minima associated with the even-order HFP modes are produced. The increase of incident angle can also reduce the electromagnetic pressure [50].

\subsection{Transition from SFP to FP resonance}

Our discussion has also been restricted to the small spacer thickness $t(t \ll h)$, which is sufficient for the SFP resonances. However, one may ask, what will happen when the spacer thickness is large? To answer the question, Figure 12 plot the reflection spectrum with the spacer thickness varying from $20 \mathrm{~nm}$ to $3 \mu \mathrm{m}(h=100 \mathrm{~nm})$. When $t$ is small, two SFP resonances can be seen in the spectrum clearly. When $t>1 \mu \mathrm{m}$, new resonant reflection minima appear, which redshift with the wavelength scaling with the spacer thickness. The resonances are just the common (vertical) FP resonance in the spacer defined by the two metal reflectors. This point can be

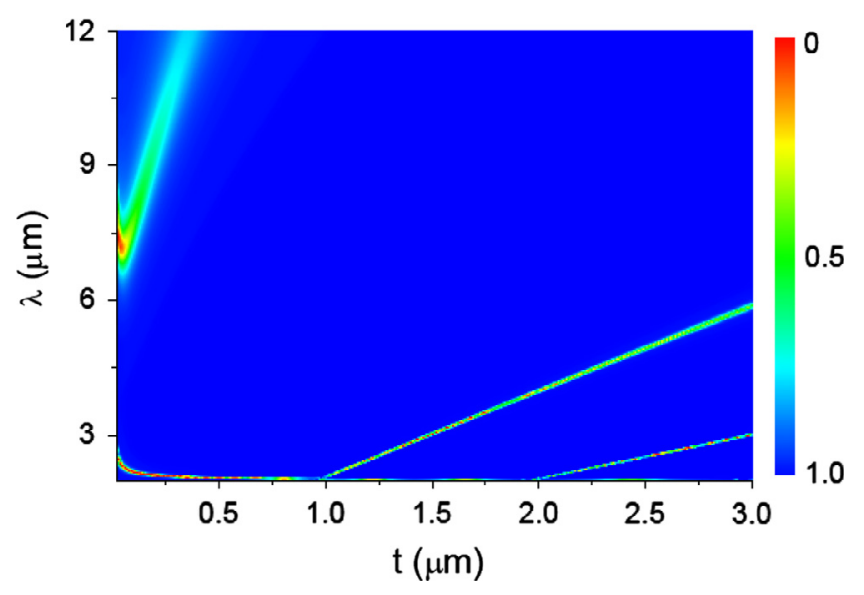

Figure 12. Reflection spectrum as a function of wavelength and spacer thickness, where a transition from the SFP resonance (in the slits) to the common FP resonance (in the spacer) is present. Here, $d=2000 \mathrm{~nm}, a=50 \mathrm{~nm}$, and $h=100 \mathrm{~nm}$.

seen with the factor $\mu_{m}$, where, for the very larger $t, \mu_{m} \approx$ $i / \tan \left(k_{0} v_{m} t\right)$. Thus, the vertical FP resonances can be induced when $k_{0} v_{0} t=m \pi$ or $2 t=m \lambda$. Such resonance can also generate an enhanced (positive) electromagnetic pressure, but the magnitude and spectral width are significantly smaller than that of SFP resonance.

\subsection{Resonances in visible/near-infrared band}

For the metal thickness or slit length much larger than the skin depth, the SFP resonances usually locate in the mid-infrared or even long wavelength regime. To conclude, we relax the constraint in the "SFP" and explore the properties of the structure in the visible or near-infrared region. Figure 13a presented the reflection spectrum of the structure, where $d=600 \mathrm{~nm}$, $a=40 \mathrm{~nm}, h=155 \mathrm{~nm}$, and $t=20 \mathrm{~nm}$. Above the Rayleigh anomaly, two reflection minima locating around the wavelength $660 \mathrm{~nm}$ and $1064 \mathrm{~nm}$ are induced. Simultaneously, strongly enhanced negative pressure can also be found at the resonance. Figure $13 \mathrm{~b}$ plots the phase shift at the slit opening as a function of wavelength. One can see that the reflection dips appear very close to the SPP resonance modes above or below the grating. Considering that the dip position is slightly modified by the slit length $h$, the resonance effect may be treated as a mixture of surface mode and slit cavity mode. However, the surface mode plays a dominant role in this case. The resonance effect in the visible or near-infrared regime may be boosted by using the laser light.

\section{Conclusions}

In summary, a special kind of deep subwavelength FP resonance in a plasmonic structure has been discussed. The plasmonic system consists of a metallic slit grating backed with a metallic ground plane. It is crucial that the structure can present a unique phase shift at the bottom slit openings, which varies gently from the positive to negative values by crossing zero. 

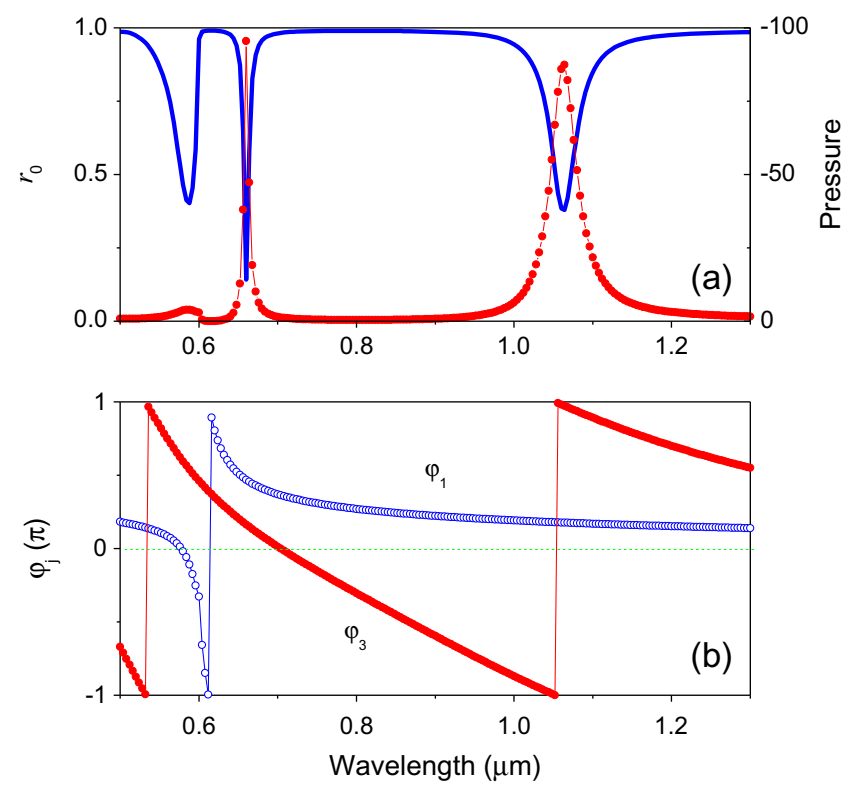

Figure 13. (a) Reflection spectrum (the line) and enhanced pressure (the circles) in the visible and near-infrared region; (b) Phase shift as a function of wavelength. Here $d=600 \mathrm{~nm}, a=40 \mathrm{~nm}$, $h=155 \mathrm{~nm}$, and $t=20 \mathrm{~nm}$. The resonance is mainly due to the grating surface mode rather than the SFP effect.

This phase shift is correlated with both the evanescent effect of diffraction components and the cavity effect of the spacer sandwiched between the two metals. The negative phase shift can compensate the positive phase accumulation in the slit cavity, thus forming, typically, a zero-order and deep subwavelength FP resonance, where the cavity length may be two orders of magnitude smaller than the resonance wavelength. By scaling the structural sizes with the wavelength, the effect can operate from the microwave band to the $\mathrm{THz}$ and infrared regime.

The SFP resonance can also induce an enhanced electromagnetic pressure. In the plasmonic system, the positive and negative forces depend on the magnetic and electric energy stored in the spacer, and a competition between them determines the sign and amplitude of the total pressure. In addition to the kinetic energy of free electrons, the "leakage" of electromagnetic energy from the spacer to the SFP resonators can modulate the total pressure. In the microwave band, the electron kinetic energy can be neglected. The "leakage" of electric (or magnetic) energy at the SFP resonances results in strong positive (or negative) pressure. The effect is similar in the $\mathrm{THz}$ band, but the electron energy begins to play a role. In the infrared regime, however, the kinetic energy of free electrons may surpass the magnetic energy and plays a crucial role. Consequently, the pressure becomes negative.

The phase shift of slit mode and the SFP resonance may be manipulated with the improved structures or designs. One can introduce a quasi-periodic rather than periodic sequence of slits to the structure, which may generate multiple SFP resonances. In addition, due to the dependence of phase shift on the slit width, the spectral width of SFP resonance can be widened by using the slits with varying slit width. This can be achieved by varying the slit width gradually along the slit array direction (the slit width is homogeneous in single slits) or employing the fan-shaped slits with the inhomogeneous width in each slit (or with the inhomogeneous lattice period). On the other hand, the SFP resonance can be manipulated dynamically by varying the incident angle or the spacer thickness. For example, by connecting the planar metal film with a piezoelectric crystal, the airspacer thickness and SFP resonance can be controlled with an external electric field. It should also be noticed that one may fill the spacer with the specific materials and control the SFP resonance using the thermal, optical, electric, or magnetic methods.

In addition to the reflection-phase modulation, ultrasmall FP resonators can also be constructed by using a cavity-index design. For example, a combination of positive and negative index materials sandwiched between two mirrors may function as an ultrasmall cavity $[92,93]$. On the other hand, the ultrasmall FP resonators can be realized with the high-index materials. For example, the indefinite medium with a unique hyperbolic dispersion can provide a larger $k$-vector or high refractive index [94]. In this case, the cavity size can be much smaller than the vacuum wavelength (but not the wavelength in the cavity) $[95,96]$. Currently, the reflection-phase and cavityindex designs for the compact FP resonators are employed independently. It may be interesting and simultaneously a challenge to consider both designs in an FP cavity. This may represent a further breakthrough towards the deep subwavelength FP resonators. A possible and simple scheme is to insert the high-index metameterials into a cavity, which is composed of the metamaterial reflectors. This would be feasible in the low frequencies such as the $\mathrm{THz}$ or microwave band.

\section{Implications and influences}

The results provide a method for designing ultrasmall FP resonators. Since no microstructured reflectors are required at the cavity opening, the cavity can be squeezed to small dimensions and operate at high frequencies. The SFP resonance may find applications in constructing ultrasensitive sensors. For example, by measuring the spectral shift of SFP resonance, one can detect the tiny motion or oscillation of ground plane with high accuracy. The SFP resonance is also accompanied by strong absorption and field-enhancement, which may boost the light-matter interactions, such as enhancing the molecule fluorescence and optical nonlinearity. In addition, the enhanced electromagnetic force can be employed to enhance the optomechanic coupling effect. With the focused and pulsed incident wave, mechanical motions of the devices or elastic waves can be excited efficiently. The SFP resonance effect may also be extended to the acoustic regime.

Acknowledgements. The authors would like to thank Dr. X.G. Yin, J.M. Zhao, and Prof. Y.J. Feng for their help in the microwave measurements. This work was supported by the Hong Kong Research Grants Council (Grant No. AOE/P-02/12), the National Basic Research Program of China (Grant No. 2012CB921502), HKUST internal (Grant No. SRFI11SC07), and the National Natural Science Foundation of China (Grant No. 11174146). 


\section{References}

1. E. Yablonovitch, Phys. Rev. Lett. 58 (1987) 2059.

2. K.M. Ho, C.T. Chan, C.M. Soukoulis, Phys. Rev. Lett. 65 (1990) 3152.

3. F.R. Montero de Espinosa, E. Jimenez, M. Torres, Phys. Rev. Lett. 80 (1998) 1208.

4. R. Sainidou, N. Stefanou, A. Modinos, Phys. Rev. B 66 (2002) 212301.

5. Y.Q. Lu, et al., Science 284 (1999) 1822.

6. Y.Y. Zhu, et al., Phys. Rev. Lett. 90 (2003) 053903.

7. C.P. Huang, Y.Y. Zhu, AIP Advances 2 (2012) 042117.

8. Z. Liu, et al., Science 289 (2000) 1734.

9. G. Wang, et al., Phys. Rev. Lett. 93 (2004) 154302.

10. W.A. Murray, W.L. Barnes, Adv. Mat. 19 (2007) 3771.

11. E. Ozbay, Science 311 (2006) 189.

12. J.A. Schuller, et al., Nat. Mater. 9 (2010) 193.

13. D.K. Gramotnev, S.I. Bozhevolnyi, Nat. Photon. 4 (2010) 83.

14. V.M. Shalaev, Nat. Photon. 1 (2007) 41.

15. X. Zhang, Z. Liu, Nat. Mater. 7 (2008) 435.

16. S. Xi, et al., Phys. Rev. Lett. 103 (2009) 194801.

17. B. Min, et al., Nature 457 (2009) 455.

18. K.J. Vahala, Nature 424 (2003) 839.

19. Y. Akahane, et al., Nature 425 (2003) 944.

20. J.B. Pendry, et al., IEEE Trans. Microwave Theor. Tech. 47 (1999) 2075.

21. T.J. Yen, et al., Science 303 (2004) 1494.

22. S. Linden, et al., Science 306 (2004) 1351.

23. C.P. Huang, et al., Phys. Rev. Lett. 104 (2010) 016402.

24. N. Engheta, A. Salandrino, A. Alù, Phys. Rev. Lett. 95 (2005) 095504

25. V. Delgado, et al., Metamaterials 3 (2009) 57.

26. C.P. Huang, et al., Opt. Express 17 (2009) 6407.

27. M. Staffaroni, et al., Photon. Nanostruct. Fund. Appl. 10 (2012) 166.

28. J. Zhou, et al., Phys. Rev. Lett. 95 (2005) 223902.

29. S. Zhang, et al., Phys. Rev. Lett. 101 (2008) 047401.

30. C. Fabry, A. Perot, Ann. Chim. Phys. 16 (1899) 115.

31. J.M. Vaughan, The Fabry-Perot interferometer: history, theory, practice, and applications, Adam Hilger, Bristol, England, 1989.

32. A. Kumar, H.D. Hristov, Microwave cavity antennas, Artech House, Norwood, MA, 1989.

33. T.J. Kippenberg, K.J. Vahala, Opt. Express 15 (2007) 17172.

34. B. Hou, et al., Phys. Rev. B 76 (2007) 054303.

35. J.A. Porto, F.J. Garcia-Vidal, J.B. Pendry, Phys. Rev. Lett. 83 (1999) 2845.

36. V.J. Sorger, et al., Nano Lett. 9 (2009) 3489.

37. A. Artar, A.A. Yanik, H. Altug, Appl. Phys. Lett. 95 (2009) 051105.

38. A. Chandran, et al., Phys. Rev. B 85 (2012) 085416.

39. V.I. Belotelov, et al., J. Opt. Soc. Am. B 29 (2012) 294.

40. R. Ameling, H. Giessen, Nano Lett. 10 (2010) 4394.

41. R. Ameling, H. Giessen, Laser \& Photon. Rev. 7 (2013) 141.

42. G.V. Trentini, IRE Trans. Antennas Propag. 53 (1956) 666.

43. D. Sievenpiper, et al., IEEE Trans. Microwave Theor. Tech. 47 (1999) 2059.
44. M.J. Lockyear, A.P. Hibbins, J.R. Sambles, Phys. Rev. Lett. 102 (2009) 073901.

45. L. Zhou, et al., Appl. Phys. Lett. 86 (2005) 101101.

46. H.Q. Li, et al., Appl. Phys. Lett. 89 (2006) 104101.

47. A. Ourir, A. de Lustrac, J. Lourtioz, Appl. Phys. Lett. 88 (2006) 084103.

48. F. Costa, A. Monorchio, Progress Electromagn. Res. 111 (2011) 467.

49. C.P. Huang, et al., Phys. Rev. B 85 (2012) 235410.

50. C.P. Huang, et al., Phys. Rev. B 86 (2012) 085446.

51. J. Li, G. Sun, C.T. Chan, Phys. Rev. B 73 (2006) 075117.

52. B. Auguie, W.L. Barnes, Phys. Rev. Lett. 101 (2008) 143902.

53. J.B. Pendry, L. Martin-Moreno, F.J. Garcia-Vidal, Science 305 (2004) 847.

54. F.J. Garcia-Vidal, L. Martin-Moreno, J.B. Pendry, J. Opt. A: Pure Appl. Opt. 7 (2005) S97.

55. J.M. Hao, L. Zhou, C.T. Chan, Appl. Phys. A 87 (2007) 281.

56. H. Raether, Surface plasmons on smooth and rough surfaces and on gratings, Springer-Verlag, New York, 1988.

57. C. Girard, A. Dereux, Rep. Prog. Phys. 59 (1996) 657.

58. L. Salomon, F. de Fornel, P.M. Adam, J. Opt. Soc. Am. A 16 (1999) 2695.

59. A.V. Zayats, I.I. Smolyaninov, A.A. Maradudin, Phys. Rep. 408 (2005) 131.

60. C.P. Huang, et al., Appl. Phys. Lett. 93 (2008) 081917.

61. T. López-Rios, et al., Phys. Rev. Lett. 81 (1998) 665.

62. Y. Takakura, Phys. Rev. Lett. 86 (2001) 5601.

63. A.P. Hibbins, M.J. Lockyear, J.R. Sambles, J. Appl. Phys. 99 (2006) 124903.

64. A.P. Hibbins, et al., Phys. Rev. Lett. 92 (2004) 143904.

65. J.R. Brown, et al., J. Appl. Phys. 104 (2008) 043105.

66. M.J. Lockyear, et al., Appl. Phys. Lett. 94 (2009) 041913.

67. Y. Todorov, et al., Phys. Rev. Lett. 102 (2009) 186402.

68. Y. Todorov, et al., Opt. Express 18 (2010) 13886.

69. P. Jouy, et al., Appl. Phys. Lett. 98 (2011) 021105.

70. Z. Wei, et al., Opt. Express 18 (2010) 12119.

71. Y.Q. Ye, Y. Jin, S.L. He, J. Opt. Soc. Am. B 27 (2010) 498.

72. J. Hao, L. Zhou, M. Qiu, Phys. Rev. B 83 (2011) 165107.

73. H.T. Chen, Opt. Express 20 (2012) 7165.

74. Y.G. Ma, C.K. Ong, X.S. Rao, J. Appl. Phys. 103 (2008) 123510.

75. N. Mattiucci, et al., Appl. Phys. Lett. 101 (2012) 141115.

76. J. Chen, et al., Nat. Photon. 5 (2011) 531.

77. A. Ashkin, Optical trapping and manipulation of neutral particles using lasers, World Scientific, Singapore, 2006.

78. A. Schliesser, et al., Phys. Rev. Lett. 97 (2006) 243905.

79. T.J. Kippenberg, K.J. Vahala, Science 321 (2008) 1172.

80. M. Eichenfield, et al., Nature 459 (2009) 550.

81. D. Van Thourhout, J. Roels, Nat. Photon. 4 (2010) 211.

82. H. Xu, M. Kall, Phys. Rev. Lett. 89 (2002) 246802.

83. L. Huang, O.J.F. Martin, Opt. Lett. 33 (2008) 3001.

84. J. Kohoutek, et al., Nano Lett. 11 (2011) 3378.

85. R. Sainidou, F.J. Garcia de Abajo, Phys. Rev. Lett. 101 (2008) 136802.

86. X. Yang, et al., Nano Lett. 11 (2011) 321.

87. D. Woolf, M. Loncar, F. Capasso, Opt. Express 17 (2009) 19996. 
88. J. Zhang, K.F. MacDonald, N.I. Zheludev, Phys. Rev. B 85 (2012) 205123.

89. H. Liu, et al., Phys. Rev. Lett. 106 (2011) 087401.

90. S.B. Wang, et al., Phys. Rev. B 84 (2011) 075114.

91. Y. Zhang, M. Han, C.P. Huang, Europhys. Lett. 102 (2013) 34001 .
92. N. Engheta, IEEE Antennas Wirel. Propag. Lett. 1 (2002) 10.

93. L. Shen, S.L. He, S. Xiao, Phys. Rev. B 69 (2004) 115111.

94. D.R. Smith, D. Schurig, Phys. Rev. Lett. 90 (2003) 077405.

95. X. Yang, et al., Nat. Photon. 6 (2012) 450.

96. J. Yao, et al., PNAS 108 (2011) 11327.

Cite this article as: Huang C-P \& Chan C-T: Deep subwavelength Fabry-Perot resonances. EPJ Appl. Metamat. $2014,1,2$. 